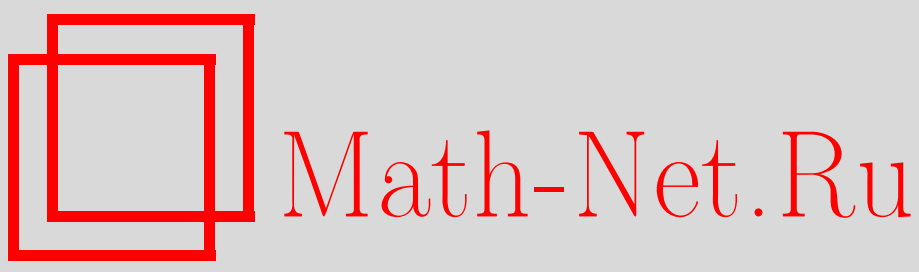

А. Ю. Колесов, Н. Х. Розов, Существование счетного числа устойчивых циклов у обобщенного кубического уравнения Шрёдингера в плоской области, Изв. РАН. Сер. матем., 2003, том 67, выпуск 6, 137-168

DOI: https://doi.org/10.4213/im462

Использование Общероссийского математического портала Math-Net.Ru подразумевает, что вы прочитали и согласны с пользовательским соглашением

http://www.mathnet.ru/rus/agreement

Параметры загрузки:

IP: 54.162 .127 .20

26 апреля 2023 г., 05:05:04 
УДК 517.926

А. Ю. Колесов, Н. Х. Розов

\section{Существование счетного числа устойчивых циклов у обобщенного кубического уравнения Шрёдингера в плоской области}

$$
\begin{gathered}
\text { В области } \Omega=\{(x, y): 0 \leqslant x \leqslant 1,0 \leqslant y \leqslant 1\} \text { рассмотрена краевая задача } \\
u_{t}+i \Delta u=\varepsilon\left(u-d|u|^{2} u\right),\left.\quad u\right|_{\partial \Omega}=0
\end{gathered}
$$

где $u$ - комплекснозначная функция, $\Delta$ - оператор Лапласа, $0<\varepsilon \ll 1, d=$ $1+i c_{0}, c_{0} \in \mathbb{R}$. Установлено существование счетного числа устойчивых периодических по $t$ решений этой задачи. Исследован вопрос о сохранении данного феномена при изменении области или граничных условий.

Библиография: 17 наименований.

\section{Введение}

K настояшему времени задача об аттракторах нелинейных гиперболических уравнений в локальной постановке в случае одной пространственной переменной изучена достаточно подробно (см. монографию [1] и последующие обзорные публикации [2]-[4]). Анализ различных математических моделей (главным образом, радиофизики) показал, что даже устойчивые двумерные торы наблюдаются весьма редко, т. е., как правило, имеются устойчивые циклы, количество которых за счет подходяшего выбора параметров может быть сколь угодно большим (это явление получило специальное название - буферность). Более того, в некоторых случаях реализуется даже гипербуферность, т. е. существует счетное число устойчивых циклов.

Явление гипербуферности было обнаружено сначала в одной конкретной гиперболической краевой задаче [5], а подробный его анализ проведен в статье [6], где дан новый, усиленный вариант определения дисперсии, отличный от общепринятого [7], и показано, что гипербуферность характерна именно для уравнений с сильной дисперсией (в [6] подробно проанализированы два типовых примера: уравнение Кортевега-де Фриса и уравнение колебаний балки).

Ниже на примере обобщенного кубического уравнения Шрёдингера исследуется новый в данной тематике вопрос о сохранении явления гипербуферности при переходе от отрезка к плоской области. Показывается, что ответ на него в зависимости от рассматриваемой области и типа граничных условий может быть как положительным, так и отрицательным.

Работа выполнена при финансовой поддержке Российского фонда фундаментальных исследований (грант № 02-01-01286).

(C) А.ю. Колесов, н.Х. Розов, 2003 


\section{§1. Уравнение Шрёдингера с граничными условиями Дирихле}

1.1. Постановка задачи. Кубическим уравнением Шрёдингера принято называть систему двух уравнений, которая в комплексной форме записи имеет вид

$$
i u_{t}=\sigma_{0} \Delta u+c_{0}|u|^{2} u,
$$

где $u$ - комплекснозначная функция, $\Delta$ - оператор Лапласа, $\sigma_{0}$ и $c_{0}$ - вещественные постоянные, причем $\sigma_{0}>0$, а знак $c_{0}$ произволен. Уравнение (1.1) возникает во многих задачах нелинейной оптики и гидродинамики [8]-[10], в частности в теории волн конечной амплитуды на поверхности глубокой жидкости. В случаях, когда необходимо учитывать диссипативные процессы, источники и стоки, это уравнение нуждается в обобшении. Простейшим из таких обобшений служит уравнение

$$
i u_{t}=\sigma_{0} \Delta u+i u+\left(c_{0}-i\right)|u|^{2} u
$$

получающееся из (1.1) при добавлении слагаемого $i u$, описывающего "подкачку энергии", и слагаемого $-i|u|^{2} u$, характеризующего нелинейную диссипацию.

Следует отметить, что (1.2) - это частньй случай известного уравнения Гинзбурга-Ландау, в правой части которого учтена дифракция (слагаемое $\sigma_{0} \Delta u$ ), но отсутствует диффузионньй член $i a \Delta u, a>0$. Такая ситуация характерна для лазерных резонаторов и других нелинейных оптических сред (см. [11]). Уравнение (1.2) описывает в этом случае пространственную эволюцию электромагнитного поля в указанных средах.

Предположим, что функция $u=u(t, x, y)$ из (1.2) зависит от двух пространственных переменных $x, y$, принадлежаших единичному квадрату $\Omega$, и удовлетворяет нулевым граничным условиям Дирихле. В результате получим краевую задачу

$$
u_{t}+i \sigma_{0} \Delta u=u-d|u|^{2} u,\left.\quad u\right|_{x=0}=\left.u\right|_{x=1}=\left.u\right|_{y=0}=\left.u\right|_{y=1}=0,
$$

где $d=1+i c_{0}$, которая и является основным объектом дальнейшего анализа. Помимо того, что эта краевая задача имеет уже упоминавшиеся физические приложения, она представляет интерес как простейшая феноменологическая модель явления диффузионной буферности, возникающего в экологии [12], [13].

Рассмотрим вопрос о сушествовании и устойчивости так называемых автомодельных циклов краевой задачи (1.3), т. е. периодических по $t$ решений вида

$$
u=u_{0}(x, y) \exp \left(i \alpha_{0} t\right), \quad \alpha_{0}=\text { const } \in \mathbb{R} .
$$

При этом устойчивость будем понимать в метрике фазового пространства (пространства начальных условий $(\operatorname{Re} u, \operatorname{Im} u)) \stackrel{\circ}{{ }_{2}^{2}} \underset{2}{2}\left(\Omega ; \mathbb{R}^{2}\right)$, где $\stackrel{\circ}{W} \underset{2}{2}-$ замыкание в метрике $W_{2}^{2}$ линеала гладких вектор-функций, удовлетворяюших граничным условиям из (1.3).

В случае одной пространственной переменной сформулированная проблема изучена достаточно подробно. В работах [1], [13] было доказано существование для аналогичной (1.3) краевой задачи

$$
u_{t}+i \sigma_{0} u_{x x}=u-d|u|^{2} u,\left.\quad u_{x}\right|_{x=0}=\left.u_{x}\right|_{x=\pi}=0
$$


счетного числа высокомодовых циклов

$$
u=u_{n}(x) \exp \left(i \alpha_{n} t\right), \quad n \geqslant n_{0} .
$$

Здесь натуральное $n_{0}$ достаточно велико и при $n \rightarrow \infty$ справедливы асимптотические равенства

$$
\alpha_{n}=\sigma_{0} n^{2}-c_{0}+O\left(\frac{1}{n^{2}}\right), \quad u_{n}(x)=\frac{2}{\sqrt{3}} \cos (n x)+O\left(\frac{1}{n^{2}}\right),
$$

второе из которых выполнено равномерно по $x$. Было установлено также, что все циклы (1.6), (1.7) экспоненциально орбитально устойчивы (неустойчивы) при $\sigma_{0}-\frac{2}{3} c_{0}>0(<0)$.

Для решения интересуюшего нас вопроса в случае краевой задачи (1.3) предположим, что $\sigma_{0} \gg 1$. После замены $\sigma_{0} t \rightarrow t$ и деления на $\sigma_{0}$ задача (1.3) преобразуется к квазилинейному виду

$$
u_{t}+i \Delta u=\varepsilon\left(u-d|u|^{2} u\right),\left.\quad u\right|_{x=0}=\left.u\right|_{x=1}=\left.u\right|_{y=0}=\left.u\right|_{y=1}=0
$$

где $\varepsilon=1 / \sigma_{0} \ll 1$, позволяющему применить к ней стандартные асимптотические методы из [14].

1.2. Теоремы о существовании автомодельных циклов. Нетрудно заметить, что линейная краевая задача, получающаяся из (1.8) при $\varepsilon=0$, допускает волновые решения

$$
u=\exp \left(i \omega_{n, k} t\right) e_{n, k}(x, y), \quad u=\exp \left(i \omega_{n, k} t\right) e_{k, n}(x, y),
$$

где

$$
\omega_{n, k}=\pi^{2}\left(n^{2}+k^{2}\right), \quad e_{n, k}(x, y)=2 \sin (n \pi x) \sin (k \pi y), \quad n, k=1,2, \ldots
$$

Поэтому естественным образом возникает вопрос о существовании у задачи (1.8) автомодельных циклов $(1.4)$ с близкими к $\omega_{n, k}$ частотами, т. е. циклов вида

$$
u=u_{n, k}(x, y, \varepsilon) \exp \left[i \alpha_{n, k}(\varepsilon) t\right], \quad \alpha_{n, k}(0)=\omega_{n, k} .
$$

Разрешение этого вопроса сводится, очевидно, к нахождению комплекснозначной амплитуды $u_{n, k}$ и вешественной постоянной $\alpha_{n, k}$ из нелинейной краевой задачи

$$
\begin{gathered}
i\left(\Delta u_{n, k}+\alpha_{n, k} u_{n, k}\right)=\varepsilon\left(u_{n, k}-d\left|u_{n, k}\right|^{2} u_{n, k}\right), \\
\left.u_{n, k}\right|_{x=0}=\left.u_{n, k}\right|_{x=1}=\left.u_{n, k}\right|_{y=0}=\left.u_{n, k}\right|_{y=1}=0 .
\end{gathered}
$$

В первую очередь рассмотрим автомодельные циклы (1.11) с номерами $n$ и $k$, удовлетворяющими требованиям

$$
n \neq k, \quad n^{2}+k^{2} \neq m^{2}+s^{2}
$$


при любых натуральных $m, s$ таких, что $(m, s) \neq(n, k),(k, n)$. Асимптотику таких циклов будем строить в виде рядов

$$
\alpha_{n, k}=\omega_{n, k}+\sum_{j=1}^{\infty} \varepsilon^{j} \alpha_{j}, \quad u_{n, k}=u_{n, k}^{0}(x, y)+\sum_{j=1}^{\infty} \varepsilon^{j} u_{n, k}^{j}(x, y)
$$

где

$$
u_{n, k}^{0}(x, y)=\xi_{1}^{0} e_{n, k}(x, y)+\xi_{2}^{0} e_{k, n}(x, y),
$$

при этом произвольные комплексные амплитуды $\xi_{j}^{0}, j=1,2$, подлежат определению. Отметим, что наличие в (1.15) двух амплитуд связано с симметрией квадрата, так как в силу равенства $\omega_{n, k}=\omega_{k, n}$ и наложенных на $n, k$ условий $(1.13)$ одной и той же частоте $\omega_{n, k}$ отвечают два линейно независимых периодических решения (1.9).

Подставляя ряды (1.14) в краевую задачу (1.12) и приравнивая коэффициенты при $\varepsilon$, приходим к линейной неоднородной задаче

$$
L u_{n, k}^{1}=f_{1}(x, y),\left.\quad u_{n, k}^{1}\right|_{x=0, x=1}=\left.u_{n, k}^{1}\right|_{y=0, y=1}=0
$$

в которой $L=i\left(\Delta+\omega_{n, k}\right), f_{1}=\left(1-i \alpha_{1}-d\left|u_{n, k}^{0}\right|^{2}\right) u_{n, k}^{0}$. Далее, из условий ее разрешимости, имеюших вид

$$
\int_{0}^{1} \int_{0}^{1} f_{1}(x, y) e_{n, k}(x, y) d x d y=\int_{0}^{1} \int_{0}^{1} f_{1}(x, y) e_{k, n}(x, y) d x d y=0
$$

для определения фигурируюших в $(1.14),(1.15)$ постоянных $\alpha_{1}, \xi_{1}^{0}, \xi_{2}^{0}$ выводим нелинейную систему

$$
\begin{aligned}
& \left(1-i \alpha_{1}\right) \xi_{1}^{0}-d\left[\frac{9}{4}\left|\xi_{1}^{0}\right|^{2} \xi_{1}^{0}+2\left|\xi_{2}^{0}\right|^{2} \xi_{1}^{0}+\left(\xi_{2}^{0}\right)^{2} \bar{\xi}_{1}^{0}\right]=0 \\
& \left(1-i \alpha_{1}\right) \xi_{2}^{0}-d\left[\frac{9}{4}\left|\xi_{2}^{0}\right|^{2} \xi_{2}^{0}+2\left|\xi_{1}^{0}\right|^{2} \xi_{2}^{0}+\left(\xi_{1}^{0}\right)^{2} \bar{\xi}_{2}^{0}\right]=0
\end{aligned}
$$

Полученная система используется при решении проблемы сушествования автомодельных циклов (1.11) с близкой к $\omega_{n, k}$ частотой, поскольку разным ее решениям отвечают циклы с различными нулевыми приближениями (1.15). Заметим далее, что при $\alpha_{1}=-c_{0}$ система (1.18) допускает, к примеру, решения $\xi_{1}^{0}=\xi_{2}^{0}=2 / \sqrt{21}$ и $\xi_{1}^{0}=2 / \sqrt{13}, \xi_{2}^{0}= \pm 2 i / \sqrt{13}$. Однако всюду ниже предполагается, что в качестве ее решения взят набор

$$
\alpha_{1}=-c_{0}, \quad \xi_{1}^{0}=2 / \sqrt{13}, \quad \xi_{2}^{0}=2 i / \sqrt{13}
$$

(причины такого выбора поясним далее).

После определения параметров $\alpha_{1}, \xi_{1}^{0}, \xi_{2}^{0}$ посредством равенств (1.19) краевая задача (1.16) становится разрешимой, а ее решение задается формулой

$$
u_{n, k}^{1}(x, y)=u_{n, k}^{1,0}(x, y)+\xi_{1}^{1} e_{n, k}(x, y)+\xi_{2}^{1} e_{k, n}(x, y) .
$$


Здесь $u_{n, k}^{1,0}$ - единственное решение краевой задачи (1.16), удовлетворяюшее условиям

$$
\int_{0}^{1} \int_{0}^{1} u_{n, k}^{1,0}(x, y) e_{n, k}(x, y) d x d y=\int_{0}^{1} \int_{0}^{1} u_{n, k}^{1,0}(x, y) e_{k, n}(x, y) d x d y=0
$$

а $\xi_{1}^{1}, \xi_{2}^{1}$ - произвольные комплексные постоянные.

В общем случае на шаге с номером $j, j \geqslant 2$, для $u_{n, k}^{j}(x, y)$ получаем аналогичную (1.16) линейную неоднородную краевую задачу

$$
L u_{n, k}^{j}=f_{j}(x, y),\left.\quad u_{n, k}^{j}\right|_{x=0, x=1}=\left.u_{n, k}^{j}\right|_{y=0, y=1}=0 .
$$

Выполнения условий ее разрешимости, имеюших вид (1.17), достигаем за счет введения очередной поправки $\alpha_{j}$ к частоте, а также слагаемого

$$
\xi_{1}^{j-1} e_{n, k}(x, y)+\xi_{2}^{j-1} e_{k, n}(x, y),
$$

с точностью до которого определяется $u_{n, k}^{j-1}$ (см. (1.20)). Следует, однако, заметить, что здесь в отличие от (1.18) для нахождения $\xi_{1}^{j-1}, \xi_{2}^{j-1}, \alpha_{j}$ возникает линейная неоднородная система вида

$$
B v=\alpha_{j} v_{0}+\psi_{j}
$$

где

$$
\begin{aligned}
& v=\operatorname{colon}\left(\xi_{1}^{j-1}, \bar{\xi}_{1}^{j-1}, \xi_{2}^{j-1}, \bar{\xi}_{2}^{j-1}\right), \quad v_{0}=\frac{2}{\sqrt{13}} \operatorname{colon}(i,-i,-1,-1), \\
& B=\left(\begin{array}{cc}
B_{1}^{-} & B_{2} \\
B_{2} & B_{1}^{+}
\end{array}\right), \quad B_{1}^{ \pm}=\left(\begin{array}{cc}
-d & \pm \frac{5}{13} d \\
\pm \frac{5}{13} \bar{d} & -\bar{d}
\end{array}\right), \quad B_{2}=\left(\begin{array}{cc}
0 & -\frac{8 i}{13} d \\
\frac{8 i}{13} \bar{d} & 0
\end{array}\right),
\end{aligned}
$$

а $\psi_{j}=\operatorname{colon}\left(\psi_{j}^{1}, \bar{\psi}_{j}^{1}, \psi_{j}^{2}, \bar{\psi}_{j}^{2}\right)$ - некоторый уже известньй вектор.

Из явного вида вектора $v_{0}$ и матрицы $B$ (см. (1.24), (1.25)) вытекает, что $B v_{0}=0$, а остальные три собственных значения этой матрицы имеют отрицательные действительные части. Следовательно, система (1.23) оказывается вырожденной, и ее разрешимость достигается за счет выбора постоянной $\alpha_{j}$ посредством равенства

$$
\alpha_{j}=-\left(\psi_{j}, w_{0}\right) .
$$

Здесь $w_{0}=(\sqrt{13} / 8) \operatorname{colon}(i d,-i \bar{d},-d,-\bar{d})$, a $(\cdot, \cdot)$ - евклидово скалярное произведение в $\mathbb{C}^{4}$ (из структуры векторов $\psi_{j}$ и $w_{0}$ следует, что правая часть формулы (1.26) всегда является вещественным числом). И наконец, после нахождения $\alpha_{j}$ решение $v$ системы (1.23), удовлетворяюшее условию $\left(v, w_{0}\right)=0$, определяется уже однозначно.

Возврашаясь к краевой задаче (1.22), заметим, что после всех проделанных построений она становится разрешимой. Решение ее задается аналогичным (1.20) равенством

$$
u_{n, k}^{j}(x, y)=u_{n, k}^{j, 0}(x, y)+\xi_{1}^{j} e_{n, k}(x, y)+\xi_{2}^{j} e_{k, n}(x, y),
$$

где функция $u_{n, k}^{j, 0}(x, y)$ удовлетворяет условиям вида (1.21), а $\xi_{1}^{j}, \xi_{2}^{j}$ - произвольные комплексные постоянные. Далее переходим к шагу с номером $j+1$, и весь процесс повторяется.

Строгий смысл изложенным построениям придает следующее утверждение. 
ТЕОРЕМа 1.1. Для любых натуральных чисел $n$ u $k$, удовлетворяющих условиям (1.13), можно указать такое достаточно малое $\varepsilon_{n, k}>0$, что при всех $0<\varepsilon \leqslant \varepsilon_{n, k}$ краевая задача (1.8) имеет автомодельный иикл (1.11), амплиmyдa

$$
u_{n, k}(x, y, \varepsilon): \quad u_{n, k}(x, y, 0)=\frac{2}{\sqrt{13}} e_{n, k}(x, y)+\frac{2 i}{\sqrt{13}} e_{k, n}(x, y)
$$

u частота

$$
\alpha_{n, k}(\varepsilon): \quad \alpha_{n, k}(0)=\omega_{n, k},\left.\quad \frac{d \alpha_{n, k}}{d \varepsilon}\right|_{\varepsilon=0}=-c_{0}
$$

которого разлагаются в сходящиеся ряды (1.14).

ДокАЗАТЕЛЬСтво. Дополним уравнение из (1.12) комплексно-сопряженным уравнением и подставим в получившуюся систему равенства

$$
\begin{gathered}
u_{n, k}=u_{n, k}^{0}+\varepsilon u_{n, k}^{1}+\varepsilon^{2} h_{1}, \quad \bar{u}_{n, k}=\bar{u}_{n, k}^{0}+\varepsilon \bar{u}_{n, k}^{1}+\varepsilon^{2} h_{2}, \\
\alpha_{n, k}=\omega_{n, k}-\varepsilon c_{0}+\varepsilon^{2} \alpha_{2},
\end{gathered}
$$

где $h_{1}, h_{2}$ - независимые комплексные переменные, функция $u_{n, k}^{0}$ определена формулами $(1.15),(1.19)$, а фигурирующие в определении $u_{n, k}^{1}$ комплексные постоянные $\xi_{1}^{1}, \xi_{2}^{1}$ (см. (1.20)) и вешественную постоянную $\alpha_{2}$ считаем произвольными. В итоге для $h=\operatorname{colon}\left(h_{1}, h_{2}\right)$ получаем уравнение вида

$$
\Pi h=G\left(x, y, h, \xi_{1}^{1}, \xi_{2}^{1}, \alpha_{2}, \varepsilon\right) .
$$

Здесь $\Pi=\operatorname{colon}(L,-L), G=\operatorname{colon}\left(G_{1}, G_{2}\right)$, оператор $L$ тот же, что и в (1.16), а компоненты вектор-функции $G$ обладают следуюшими свойствами:

$$
\begin{gathered}
G_{2}=\bar{G}_{1}^{*}, \\
\left.G_{1}\right|_{\varepsilon=0}=-i \alpha_{2} u_{n, k}^{0}+d\left(u_{n, k}^{1}-\left(u_{n, k}^{0}\right)^{2} \bar{u}_{n, k}^{1}-2 u_{n, k}^{1}\left|u_{n, k}^{0}\right|^{2}\right),
\end{gathered}
$$

где $G_{1}^{*}$ получено из $G_{1}$ при замене $h$ на $h^{*}=\operatorname{colon}\left(\bar{h}_{2}, \bar{h}_{1}\right)$.

Рассмотрим введенный вьше оператор П в инвариантном подпространстве $V_{0} \subset$ $\stackrel{\circ}{W}_{2}^{2} \times \stackrel{\circ}{W} \underset{2}{2}$ вектор-функций (с комплексными компонентами), ортогональных в смысле $L_{2} \times L_{2}$ вектор-функциям

$$
a_{j} e_{n, k}(x, y), \quad a_{j} e_{k, n}(x, y), \quad j=1,2
$$

где $a_{1}=\operatorname{colon}(1,0), a_{2}=\operatorname{colon}(0,1)$. Нетрудно видеть, что этот оператор имеет в $V_{0}$ ограниченный обратный. Поэтому, изменяя надлежашим образом неоднородность $G$ и обращая П, перейдем от $(1.30)$ к интегральному уравнению в пространстве $V_{0}$ :

$$
h=\Pi^{-1}\left(G-\gamma_{1} a_{1} e_{n, k}-\gamma_{2} a_{2} e_{n, k}-\gamma_{3} a_{1} e_{k, n}-\gamma_{4} a_{2} e_{k, n}\right),
$$


где

$$
\begin{array}{ll}
\gamma_{1}=\int_{0}^{1} \int_{0}^{1}\left(G, a_{1}\right) e_{n, k}(x, y) d x d y, & \gamma_{2}=\int_{0}^{1} \int_{0}^{1}\left(G, a_{2}\right) e_{n, k}(x, y) d x d y \\
\gamma_{3}=\int_{0}^{1} \int_{0}^{1}\left(G, a_{1}\right) e_{k, n}(x, y) d x d y, & \gamma_{4}=\int_{0}^{1} \int_{0}^{1}\left(G, a_{2}\right) e_{k, n}(x, y) d x d y .
\end{array}
$$

Заметим, далее, что правая часть уравнения (1.34) порождает в $V_{0}$ нелинейный оператор $F$, аналитически зависяший как от $h$, так и от вешественных параметров

$$
\varepsilon, \quad \alpha_{2}, \quad \operatorname{Re} \xi_{j}^{1}, \quad \operatorname{Im} \xi_{j}^{1}, \quad j=1,2
$$

Кроме того, из равенств $(1.31),(1.32)$ очевидным образом следует, что оператор $F$ преобразует в себя некоторьй шар пространства $V_{0}$ не зависяшего от $\varepsilon$ радиуса с центром в нуле и является в нем сжимающим (с константой сжатия порядка $\varepsilon$ ). Тем самым, в силу принципа сжимающих отображений, соответствующий вариант которого приведен, например, в [15], из (1.34) однозначно определяется аналитически зависящая (в метрике $V_{0}$ ) от параметров (1.36) вектор-функция

$$
h_{0}=\operatorname{colon}\left(h_{1}^{0}\left(x, y, \alpha_{2}, \xi_{1}^{1}, \xi_{2}^{1}, \varepsilon\right), h_{2}^{0}\left(x, y, \alpha_{2}, \xi_{1}^{1}, \xi_{2}^{1}, \varepsilon\right)\right)
$$

Отметим еще, что из свойства (1.31) правой части уравнения (1.34) вытекает равенство

$$
h_{2}^{0}=\bar{h}_{1}^{0}
$$

В заключение определим постоянную $\alpha_{2} \in \mathbb{R}$ и вектор $v=\operatorname{colon}\left(\xi_{1}^{1}, \bar{\xi}_{1}^{1}, \xi_{2}^{1}, \bar{\xi}_{2}^{1}\right)$, который будем предполагать ортогональным вектору $w_{0}$, из условий равенства нулю фигурирующих в (1.34) поправок к неоднородности $G$. В связи с этим подставим в правые части равенств (1.35) вектор-функцию (1.37). В результате получим аналитические по совокупности переменных (1.36) функции $\gamma_{j}\left(\varepsilon, \alpha_{2}, v\right), j=$ $1, \ldots, 4$, для которых в силу $(1.31),(1.38)$ справедливы тождества $\gamma_{2} \equiv \bar{\gamma}_{1}, \gamma_{4} \equiv \bar{\gamma}_{3}$. Тем самым проблема сводится к нахождению $\alpha_{2} \in \mathbb{R}$ и $v \in \mathbb{C}^{4},\left(v, w_{0}\right)=0$, из системы

$$
\Gamma\left(\varepsilon, \alpha_{2}, v\right)=0
$$

где $\Gamma=\operatorname{colon}\left(\gamma_{1}, \bar{\gamma}_{1}, \gamma_{3}, \bar{\gamma}_{3}\right)$.

Используя очередной раз формулы (1.31), (1.32), нетрудно убедиться в том, что при $\varepsilon=0$ система (1.39) переходит в линейную неоднородную систему вида (1.23), которая, напомним, при подходящем выборе параметра $\alpha_{2}$ (см. (1.26)) однозначно разрешима в требуемом классе векторов. Отсюда и из теоремы о неявной функции следует, что исходная система (1.39) также имеет аналитически зависящее от $\varepsilon$ решение

$$
\left(v(\varepsilon), \alpha_{2}(\varepsilon)\right): \quad\left(v(\varepsilon), w_{0}\right) \equiv 0
$$

при всех достаточно малых $\varepsilon>0$. Наконец, подставляя равенства $(1.40),(1.37)$ в (1.29), получаем аналитически зависящий от $\varepsilon$ автомодельный цикл краевой задачи (1.8) с требуемыми свойствами (1.27), (1.28). Теорема доказана. 
Следует отметить, что в силу инвариантности краевой задачи (1.8) относительно замен $x \rightarrow y, y \rightarrow x$ наряду с циклом (1.11), (1.27), (1.28) она всегда имеет ассоциированный с ним автомодельный цикл

$$
\begin{gathered}
u=u_{k, n}(x, y, \varepsilon) \exp \left[i \alpha_{k, n}(\varepsilon) t\right]: u_{k, n}(x, y, \varepsilon)=u_{n, k}(y, x, \varepsilon), \\
\alpha_{k, n}(\varepsilon)=\alpha_{n, k}(\varepsilon) .
\end{gathered}
$$

Отметим также, что циклу (1.41) в системе (1.18) соответствует решение $\alpha_{1}=-c_{0}, \xi_{1}^{0}=2 i / \sqrt{13}, \xi_{2}^{0}=2 / \sqrt{13}$.

Полученная теорема, а также принцип подобия используются для решения проблемы существования счетного числа автомодельных циклов специального вида краевой задачи (1.8). Для пояснения этого положим $n=1, k=2$. Данная пара индексов удовлетворяет, очевидно, условиям (1.13). Поэтому найдется такое достаточно малое $\varepsilon_{0}>0$, что при всех $0<\varepsilon \leqslant \varepsilon_{0}$ краевая задача (1.8) имеет пару ассоциированных автомодельных циклов

$$
u=u_{1,2}(x, y, \varepsilon) \exp \left[i \alpha_{1,2}(\varepsilon) t\right], \quad u=u_{2,1}(x, y, \varepsilon) \exp \left[i \alpha_{2,1}(\varepsilon) t\right],
$$

удовлетворяющих равенствам (1.27), (1.28).

Следующий шаг состоит в применении к циклам (1.42) принципа подобия, который, в свою очередь, разбивается на два этапа.

ЭТАП 1. Продолжим функиию $u_{1,2}(x, y, \varepsilon)$ из (1.42) по переменным $x, y$ с единичного квадрата на всю плоскость следующим образом:

а) сначала в квадрат $\{(x, y): 0 \leqslant x \leqslant 1,-1 \leqslant y \leqslant 0\}$ по правилу $u_{1,2}(x$, $-y, \varepsilon) \equiv-u_{1,2}(x, y, \varepsilon)$

б) затем в полосу $\{(x, y): 0 \leqslant x \leqslant 1, y \in \mathbb{R}\}$ по закону периодичности $u_{1,2}(x, y+2, \varepsilon) \equiv u_{1,2}(x, y, \varepsilon)$

в) далее в полосу $\{(x, y):-1 \leqslant x \leqslant 0, y \in \mathbb{R}\}$ посредством равенства $u_{1,2}(-x, y, \varepsilon) \equiv-u_{1,2}(x, y, \varepsilon)$;

г) и наконеи, на всю плоскость $(x, y) \in \mathbb{R}^{2}$ с помощью равенства $u_{1,2}(x+2$, $y, \varepsilon) \equiv u_{1,2}(x, y, \varepsilon)$.

После әтого аналогичным образом продолжим функиию $u_{2,1}(x, y, \varepsilon)$. В результате получим пару автомодельных циклов, удовлетворяющих уравнению из (1.8) при всех $(x, y) \in \mathbb{R}^{2}$.

ЭТАП 2. Введем в рассмотрение счетное семейство автомодельных ииклов

$$
\begin{gathered}
u=u_{2 k, k}(x, y, \varepsilon) \exp \left[i \alpha_{2 k, k}(\varepsilon) t\right], \\
u=u_{k, 2 k}(x, y, \varepsilon) \exp \left[i \alpha_{k, 2 k}(\varepsilon) t\right], \quad k \geqslant 1,
\end{gathered}
$$

гдe

$$
\begin{gathered}
u_{k, 2 k}=u_{1,2}\left(k x, k y, \frac{\varepsilon}{k^{2}}\right), \quad u_{2 k, k}=u_{2,1}\left(k x, k y, \frac{\varepsilon}{k^{2}}\right), \\
\alpha_{k, 2 k}=\alpha_{2 k, k}=k^{2} \alpha_{1,2}\left(\frac{\varepsilon}{k^{2}}\right) .
\end{gathered}
$$

Непосредственной проверкой убеждаемся в том, что все они являются решениями исходной краевой задачи (1.8).

Объединяя все сказанное, приходим к следующему утверждению. 
Tеорема 1.2. Пусть $\varepsilon_{0}$ - доставляемое теоремой 1.1 значение $\varepsilon_{n, k}$ при $n=1, k=2$. Тогда при всех $0<\varepsilon \leqslant \varepsilon_{0}$ краевая задача (1.8) имеет счетное число автомодельных ичиклов (1.43).

Из способа построения циклов (1.43) следует, что аналог теоремы 1.2 справедлив не только при малых $\varepsilon$, но и вообще при любом $\varepsilon>0$. Однако в этом случае можно гарантировать лишш сушествование циклов с номерами $k$, удовлетворяющими неравенству $\varepsilon / k^{2} \leqslant \varepsilon_{0}$.

1.3. Теорема об устойчивости циклов. Устойчивость построенных вьше циклов зависит от расположения собственных значений спектральных задач

$$
H_{k} z=\lambda z,\left.\quad z\right|_{x=0}=\left.z\right|_{x=1}=\left.z\right|_{y=0}=\left.z\right|_{y=1}=0
$$

где $z=\operatorname{colon}\left(z_{1}, z_{2}\right)$

$$
\begin{gathered}
H_{k}=\left(\begin{array}{rr}
-i & 0 \\
0 & i
\end{array}\right) \Delta+\left(\begin{array}{cc}
\varkappa_{1, k}(x, y, \varepsilon) & \varkappa_{2, k}(x, y, \varepsilon) \\
\varkappa_{2, k}(x, y, \varepsilon) & \varkappa_{1, k}(x, y, \varepsilon)
\end{array}\right), \quad k \geqslant 1, \\
\varkappa_{1, k}=\varepsilon\left(1-2 d\left|u_{2 k, k}\right|^{2}\right)-i \alpha_{2 k, k}, \quad \varkappa_{2, k}=-\varepsilon d u_{2 k, k}^{2} .
\end{gathered}
$$

Действительно, операторы (1.45) получаются из уравнения (1.8) и комплексно-сопряженного к нему уравнения после замен

$$
u \exp \left(-i \alpha_{k, 2 k} t\right) \rightarrow u, \quad \bar{u} \exp \left(i \alpha_{k, 2 k} t\right) \rightarrow \bar{u}
$$

переводящих в состояния равновесия $\left(u_{2 k, k}, \bar{u}_{2 k, k}\right),\left(u_{k, 2 k}, \bar{u}_{k, 2 k}\right)$ циклы $(1.43)$, и последуюшей линеаризации на первом из этих состояний равновесия (случай второго цикла (1.43) рассматривать не будем, поскольку он сводится к данному посредством замен $x \rightarrow y, y \rightarrow x)$. Отметим также, что каждый из операторов (1.45) имеет нулевое собственное значение, которому отвечает собственная функция

$$
z_{0}(x, y, \varepsilon)=\operatorname{colon}\left(i u_{2 k, k}(x, y, \varepsilon),-i \bar{u}_{2 k, k}(x, y, \varepsilon)\right) .
$$

И наконец, отметим, что если спектральная задача (1.44) имеет собственное значение $\lambda_{0}$ с собственной функцией $\operatorname{colon}\left(z_{1}^{0}, z_{2}^{0}\right)$, то собственным будет и значение $\bar{\lambda}_{0}$ с собственной функцией $\operatorname{colon}\left(\bar{z}_{2}^{0}, \bar{z}_{1}^{0}\right)$.

Теорема 1.3. Существуют такие положительные постоянные $\varepsilon_{0}$ и $\gamma_{0}$, что при всех $0<\varepsilon \leqslant \varepsilon_{0}$ и при каждом натуральном $k$ нулевое собственное значение краевой задачи (1.44) является простьм, а весь остальной ее спектр лежит в комплексной полуплоскости $\left\{\lambda: \operatorname{Re} \lambda \leqslant-\gamma_{0} \varepsilon\right\}$.

Доказательству теоремы 1.3 посвятим отдельньй параграф. Здесь же отметим, что из ее утверждения следует устойчивость при любом фиксированном достаточно малом $\varepsilon>0$ всех циклов (1.43). Тем самым в рамках краевой задачи (1.8) реализуется феномен гипербуферности. Более того, как и совокупность циклов (1.6) краевой задачи (1.5), циклы (1.43) обладают свойством равномерной устойчивости. Данное свойство заключается в том, что фигурируюшая в теореме постоянная $\gamma_{0}$ не зависит от номера $k$. 
1.4. О разрушении гипербуферности в прямоугольнике. Весьма неожиданныт оказывается тот факт, что при переходе в краевой задаче (1.8) от квадрата к прямоугольнику

$$
\Omega=\left\{(x, y): 0 \leqslant x \leqslant l_{1}, 0 \leqslant y \leqslant l_{2}\right\}
$$

с иррациональньм отношением $l_{1}^{2} / l_{2}^{2}$ феномен гипербуферности исчезает. Более того, в случае области (1.46) локальными методами не удается обнаружить ни одного устойчивого цикла или тора.

Для удобства последующего анализа выполним в уравнении из (1.8) замены $x / l_{1} \rightarrow x, y / l_{2} \rightarrow y$. В результате снова получим краевую задачу в единичном квадрате:

$$
u_{t}+i L u=\varepsilon\left(u-d|u|^{2} u\right),\left.\quad u\right|_{x=0}=\left.u\right|_{x=1}=\left.u\right|_{y=0}=\left.u\right|_{y=1}=0
$$

где $L=\sigma_{1}^{2} \partial^{2} / \partial x^{2}+\sigma_{2}^{2} \partial^{2} / \partial y^{2}, \sigma_{j}=1 / l_{j}, j=1,2$. Заметим, далее, что при $\varepsilon=0$ она допускает аналогичные (1.9) волновые решения

$$
u=\exp \left(i \omega_{n, k} t\right) e_{n, k}(x, y), \quad \omega_{n, k}=\pi^{2}\left(\sigma_{1}^{2} n^{2}+\sigma_{2}^{2} k^{2}\right), \quad n, k \geqslant 1,
$$

где для $e_{n, k}(x, y)$ сохраняются прежние формулы из (1.10). Поэтому для построения асимптотики возможных автоколебаний данной задачи воспользуемся бесконечномерным вариантом асимптотического метода Крылова-Боголюбова-Митропольского, предложенньм в [16].

Используя сушествование волновых решений (1.48), подставим в (1.47) ряд

$$
u=u_{0}(t, \tau, x, y)+\varepsilon u_{1}(t, \tau, x, y)+\cdots, \quad \tau=\varepsilon t,
$$

где

$$
u_{0}=\sum_{n, k=1}^{\infty} \xi_{n, k}(\tau) \exp \left(i \omega_{n, k} t\right) e_{n, k}(x, y),
$$

а подлежашие определению комплексные амплитуды $\xi_{n, k}$ таковы, что сходится ряд с обшим членом $\omega_{n, k}^{2}\left|\xi_{n, k}\right|^{2}$ (в этом случае $u_{0} \in \stackrel{\circ}{W_{2}^{2}}(\Omega)$ ). Приравнивая затем коэффициенты при $\varepsilon$, для нахождения $u_{1}$ получим краевую задачу

$$
\frac{\partial u_{1}}{\partial t}+i L u_{1}=g(t, \tau, x, y),\left.\quad u_{1}\right|_{x=0, x=1}=\left.u_{1}\right|_{y=0, y=1}=0
$$

в которой $g=-\partial u_{0} / \partial \tau+\left(1-d\left|u_{0}\right|^{2}\right) u_{0}$, а переменная $\tau$ рассматривается как параметр.

При анализе краевой задачи (1.51) сушественную роль играет предполагаемая иррациональность числа $\sigma_{1}^{2} / \sigma_{2}^{2}$, поскольку она исключает одновременное выполнение равенств вида

$$
\omega_{n, k}=\omega_{n_{1}, k_{1}}+\omega_{n_{2}, k_{2}}-\omega_{n_{3}, k_{3}}, \quad n=n_{1} \pm n_{2} \pm n_{3}, \quad k=k_{1} \pm k_{2} \pm k_{3}
$$


при произвольном выборе комбинаций знаков + и - во втором и третьем из них (тождественные резонансы, когда $(n, k)=\left(n_{1}, k_{1}\right),\left(n_{2}, k_{2}\right)=\left(n_{3}, k_{3}\right)$, здесь, естественно, не рассматриваются). Таким образом, функция $u_{1}$ может быть найдена из (1.51) в виде формального тригонометрического ряда переменной $t$ в том и только том случае, если в неоднородности $g(t, \tau, x, y)$ отсутствуют гармоники вида (1.48). Поэтому приравняем нулю коэффициенты при указанных гармониках. В результате для фигурирующих в (1.50) амплитуд $\xi_{n, k}$ приходим к счетной системе обыкновенных дифференциальных уравнений

$$
\begin{gathered}
\frac{d \xi_{n, k}}{d \tau}=\xi_{n, k}-d\left[\frac{9}{4}\left|\xi_{n, k}\right|^{2}+3 \sum_{\substack{m=1 \\
m \neq n}}^{\infty}\left|\xi_{m, k}\right|^{2}+3 \sum_{\substack{m=1 \\
m \neq k}}^{\infty}\left|\xi_{n, m}\right|^{2}\right. \\
\left.+2 \sum_{\substack{m, r=1 \\
m \neq n, r \neq k}}^{\infty}\left|\xi_{m, r}\right|^{2}\right] \xi_{n, k}, \quad n, k=1,2, \ldots
\end{gathered}
$$

Далее, систему для $\rho_{n, k}=\left|\xi_{n, k}\right|^{2}$, которая после замены $2 \tau \rightarrow \tau$ приобретает вид

$$
\begin{gathered}
\frac{d \rho_{n, k}}{d \tau}=\left(1-\frac{9}{4} \rho_{n, k}-3 \sum_{m \neq k} \rho_{n, m}-3 \sum_{m \neq n} \rho_{m, k}-2 \sum_{m \neq n, r \neq k} \rho_{m, r}\right) \rho_{n, k}, \\
n, k=1,2, \ldots
\end{gathered}
$$

будем рассматривать как абстрактное эволюционное уравнение в пространстве последовательностей $\rho=\left\{\rho_{n, k}, n, k=1,2, \ldots\right\}$ с нормой

$$
\|\rho\|=\sum_{n, k=1}^{\infty} \omega_{n, k}^{2}\left|\rho_{n, k}\right|<\infty .
$$

Из структуры входящих в (1.53) нелинейностей и дифференциальных неравенств

$$
\frac{d \rho_{n, k}}{d \tau} \leqslant \rho_{n, k}, \quad n, k=1,2, \ldots
$$

следует, что решение $\rho=\rho(\tau)$ этой системы с произвольным начальным условием $\rho(0)=\rho_{0} \in K_{+}$, где $K_{+}-$конус векторов с неотрицательными координатами, определено на полуоси $\tau \geqslant 0$ и остается при всех $\tau$ в конусе $K_{+}$.

Предположим, что система (1.53) имеет состояние равновесия с конечным числом положительных координат, равным $p \geqslant 1$, т. е.

$$
\rho_{n_{j}, k_{j}}=\eta_{j}>0, \quad j=1, \ldots, p, \quad \rho_{n, k}=0 \text { при }(n, k) \neq\left(n_{j}, k_{j}\right) .
$$

Справедливо следующее утверждение.

ТЕОРЕма 1.4. Любое состояние равновесия системы (1.53) вида (1.54) экспоненииально неустойчиво, причем бесконечна размерность его неустойчивого многообразия. 
ДокАЗАТЕЛЬСтво. Рассмотрим систему для нахождения компонент $\eta_{j}$ из (1.54), которая, очевидно, имеет вид

$$
\alpha_{j, 1} \eta_{1}+\alpha_{j, 2} \eta_{2}+\cdots+\alpha_{j, p} \eta_{p}=1, \quad j=1, \ldots, p,
$$

где $\alpha_{j, j}=9 / 4, j=1, \ldots, p$, а все остальные коэффициенты $\alpha_{j, k}, j \neq k$, равны 2 или 3 . Тем самым, заменяя в $(1.55)$ все $\alpha_{j, k}$ при $j \neq k$ на 2 , приходим к серии оценок

$$
2 \eta_{1}+\cdots+2 \eta_{j-1}+\frac{9}{4} \eta_{j}+2 \eta_{j+1}+\cdots+2 \eta_{p} \leqslant 1, \quad j=1, \ldots, p .
$$

Суммируя все эти неравенства, убеждаемся в том, что

$$
S \equiv \sum_{j=1}^{p} \eta_{j} \leqslant \frac{4 p}{8 p+1}
$$

Наконец, рассмотрим систему в вариациях на интересующем нас состоянии равновесия. Если заметить, что любое ее уравнение с индексами $m, r$, для которых $m \neq n_{j}$ и $r \neq k_{j}, j=1, \ldots, p$, имеет вид

$$
\frac{d h_{m, r}}{d \tau}=(1-2 S) h_{m, r}
$$

то из оценки (см. (1.56))

$$
1-2 S \geqslant \frac{1}{8 p+1}
$$

требуемый факт вытекает очевидным образом. Теорема доказана.

Нетрудно видеть, что состоянию равновесия (1.54) в системе (1.52) соответствует автомодельный $p$-мерный тор

$$
\begin{gathered}
\xi_{n_{j}, k_{j}}(\tau)=\sqrt{\eta_{j}} \exp \left(i \psi_{j} \tau\right), \quad j=1, \ldots, p, \\
\xi_{n, k}=0 \text { при }(n, k) \neq\left(n_{j}, k_{j}\right),
\end{gathered}
$$

где $\psi_{j} \in \mathbb{R}$. Далее, из равенства (1.57) можно однозначно определить первые два члена ряда (1.49). Тем самым получаем приближенный (с точностью до $\varepsilon^{2}$ по невязке) $p$-мерный инвариантный тор исходной краевой задачи (1.47). Однако, как следует из теоремы 1.4, соответствующий ему точный инвариантный тор если и существует, то заведомо неустойчив.

Вопрос об аттракторах краевой задачи (1.47) при иррациональном отношении $\sigma_{1}^{2} / \sigma_{2}^{2}$ остается открытым. Наиболее вероятной гипотезой представляется следующее: при $t \rightarrow \infty$ ее решения приобретают турбулентный характер, т. е. происходит их стохастизация по $t$ с одновременной потерей гладкости по пространственным переменным. Некоторые аргументы в пользу данной гипотезы приведены в статье [17]. 


\section{§2. Доказательство теоремы 1.3}

2.1. Исследование устойчивости в нерезонансных случаях. Приступим к изложению алгоритмической части обоснования теоремы 1.3, которая заключается в асимптотическом вычислении собственных значений краевой задачи

$$
\Pi_{k} z+\varepsilon A_{k}(x, y) z=\lambda z,\left.\quad z\right|_{x=0}=\left.z\right|_{x=1}=\left.z\right|_{y=0}=\left.z\right|_{y=1}=0
$$

где

$$
\begin{gathered}
\Pi_{k}=\operatorname{diag}\left\{-i\left(\Delta+\omega_{2 k, k}\right), i\left(\Delta+\omega_{2 k, k}\right)\right\}, \quad A_{k}(x, y)=\left(\begin{array}{cc}
\varkappa_{1, k}^{0} & \varkappa_{2, k}^{0} \\
\bar{\varkappa}_{2, k}^{0} & \bar{\varkappa}_{1, k}^{0}
\end{array}\right), \\
\varkappa_{1, k}^{0}=d\left(1-2\left|u_{2 k, k}(x, y, 0)\right|^{2}\right), \quad \varkappa_{2, k}^{0}=-d u_{2 k, k}^{2}(x, y, 0) .
\end{gathered}
$$

Отметим, что данная задача получается из исходной (1.44) в результате исключения из ее коэффишиентов несушественных для дальнейшего слагаемых, имеюших равномерно по $k \geqslant 1$ и $(x, y) \in \Omega$ порядок малости не менее $\varepsilon^{2}$.

При $\varepsilon=0$ спектр краевой задачи (2.1) ясен - это последовательность собственных значений

$$
\lambda_{m, s}^{ \pm}= \pm i\left(\omega_{m, s}-\omega_{k, 2 k}\right), \quad m, s=1,2, \ldots,
$$

которым соответствуют собственные функции $a_{1} e_{m, s}(x, y), a_{2} e_{m, s}(x, y)$, где, напомним, векторы $a_{1}, a_{2}$ определены в (1.33). Однако, поскольку наряду с комплексным собственным значением задача (2.1) всегда имеет комплексно-сопряженное значение, при некоторой обшности положения относительно $m$ и $s$ достаточно найти "поправки" порядка $\varepsilon$ лиш к группе значений (2.2), которой соответствует знак + . Исключение здесь составляют так называемые резонансные случаи, которые будут рассмотрены отдельно.

Итак, фиксируем произвольно индексы $m, s$. Асимптотики собственного значения и собственной функции спектральной задачи (2.1) с нулевыми приближениями $\lambda_{m, s}^{+}, a_{1} e_{m, s}(x, y)$ будем строить (когда это возможно) в одномодовом виде:

$$
z=a_{1} e_{m, s}(x, y)+\varepsilon z_{m, s}(x, y)+\cdots, \quad \lambda=\lambda_{m, s}^{+}+\varepsilon \mu_{m, s}+\cdots .
$$

В результате после подстановки равенств (2.3) в (2.1) и приравнивания коэффициентов при $\varepsilon$ получим краевую задачу

$$
\begin{gathered}
\left(\Pi_{k}-\lambda_{m, s}^{+} I\right) z_{m, s}=\left(\mu_{m, s} a_{1}-A_{k}(x, y) a_{1}\right) e_{m, s}(x, y), \\
\left.z_{m, s}\right|_{x=0, x=1}=\left.z_{m, s}\right|_{y=0, y=1}=0 .
\end{gathered}
$$

Анализ этой задачи стандартен: сначала, приравнивая в ее правой части нулю коэффициент при $a_{1} e_{m, s}(x, y)$, находим комплексную постоянную $\mu_{m, s}$, а затем ищем $z_{m, s}$ в виде тригонометрического полинома переменных $x, y$ той же структуры, что и получившаяся в итоге неоднородность.

Рассмотрим сначала случай, когда в $(2.3),(2.4)$

$$
(m, s)=(k, p): \quad p \geqslant 1, \quad p \neq k, 2 k
$$


Несложным подсчетом находим, что здесь, во-первых,

$$
\mu_{k, p}=-\frac{7 d}{13},
$$

во-вторых, после определения $\mu_{k, p}$ неоднородность в (2.4) становится линейной комбинацией гармоник вида

$$
a_{j} e_{r, q}(x, y), \quad j=1,2,
$$

где при $j=1$ индексы $r, q$ пробегают значения

$$
\begin{gathered}
(r, q)=(k, p \pm 4 k),(3 k, p),(3 k, p \pm 4 k),(k, p \pm 2 k), \\
(3 k, p \pm 2 k),(5 k, p \pm 2 k),(5 k, p),
\end{gathered}
$$

а при $j=2$ к этим значениям добавляются еще наборы индексов

$$
(r, q)=(2 k, p \pm k),(2 k, p \pm 3 k),(4 k, p \pm k),(4 k, p \pm 3 k),(k, p) .
$$

Поэтому для нахождения $z_{k, p}$ в виде линейной комбинации тех же функций $(2.7)$ необходимо и достаточно выполнение соответствующих условий нерезонансности

$$
\omega_{k, p}-\omega_{r, q} \neq 0, \quad 2 \omega_{k, 2 k} \neq \omega_{r, q}+\omega_{k, p}
$$

где в первом случае индексы $(r, q)$ принимают только значения $(2.8)$, а во втором все значения (2.8), (2.9).

Следует отметить, что часть неравенств (2.10) справедлива автоматически, а некоторые из них при подходящем выборе натурального $p$ могут нарушаться. Для того чтобы разобраться с данным вопросом, привлечем явные формулы для частот (см. (1.10)). В итоге убеждаемся в том, что все неравенства из второй группы (2.10) и большинство неравенств из первой группы вьполняются при каждом натуральном $p$ из (2.5). Исключение составляют только условия

$$
\omega_{k, p}-\omega_{3 k, p-4 k} \neq 0, \quad \omega_{k, p}-\omega_{3 k, p-2 k} \neq 0, \quad \omega_{k, p}-\omega_{5 k, p-2 k} \neq 0,
$$

первые два из которых нарушаются при $p=3 k$, а третье - при $p=7 k$. Поэтому предположим пока, что в (2.5)

$$
p \neq 3 k, 7 k
$$

(случаи $p=3 k$ и $p=7 k$ будут рассмотрены в следуюшем пункте).

Анализ краевой задачи (2.4) при

$$
(m, s)=(p, k): \quad p \geqslant 1, \quad p \neq k, 2 k,
$$

идентичен описанному выше. Так, например, здесь для $\mu_{p, k}$ сохраняется прежнее равенство (2.6). Далее, после определения $\mu_{p, k}$ неоднородность в (2.4) становится линейной комбинацией гармоник (2.7), в которых $r$ и $q$ следует поменять местами. Поэтому возможность определения $z_{p, k}$ в виде линейной комбинации тех же гармоник по-прежнему обеспечивают условия (2.11). 
Как и в двух предыдуших ситуациях, реализация алгоритма при

$$
(m, s)=(2 k, p),(p, 2 k): \quad p \geqslant 1, \quad p \neq k, 2 k,
$$

связана с однотипными рассмотрениями. В частности, здесь по-прежнему

$$
\mu_{2 k, p}=\mu_{p, 2 k}=-\frac{7 d}{13},
$$

а после определения, например, $\mu_{2 k, p}$ неоднородность в соответствующей краевой задаче (2.4) становится линейной комбинацией гармоник (2.7), где при $j=1$ индексы $(r, q)$ принимают значения

$$
(r, q)=(2 k, p \pm 4 k),(4 k, p),(4 k, p \pm 4 k),(2 k, p \pm 2 k),(6 k, p),(6 k, p \pm 2 k),
$$

а при $j=2$ к ним следует добавить еще значения

$$
\begin{gathered}
(r, q)=(k, p \pm k),(k, p \pm 3 k),(3 k, p \pm k),(3 k, p \pm 3 k) \\
(5 k, p \pm k),(5 k, p \pm 3 k),(2 k, p)
\end{gathered}
$$

(если же $(m, s)=(p, 2 k)$, то $r$ и $q$ в $(2.15),(2.16)$ меняются местами). Таким образом, обе функции $z_{2 k, p}$ и $z_{p, 2 k}$ могут быть найдены в виде соответствуюших линейных комбинаций гармоник (2.7) при выполнении одних и тех же условий нерезонансности

$$
\omega_{2 k, p}-\omega_{r, q} \neq 0, \quad 2 \omega_{k, 2 k} \neq \omega_{r, q}+\omega_{2 k, p},
$$

где, как и вьше, в первом из этих неравенств индексы $(r, q)$ принимают значения (2.15), а во-втором - значения $(2.15),(2.16)$.

Непосредственной проверкой убеждаемся в том, что большинство условий $(2.17)$ выполняется при всех допустимых значениях $p$ из (2.13). Исключениями являются только два неравенства

$$
\omega_{2 k, p} \neq \omega_{6 k, p-2 k}, \quad \omega_{2 k, p} \neq \omega_{4 k, p-4 k},
$$

которые нарушаются соответственно при $p=9 k$ и $p=7 k / 2$ (в последнем случае предполагается, естественно, что $k$ четное). Поэтому будем считать, что в (2.13)

$$
p \neq 9 k, \frac{7 k}{2} .
$$

Следует исключить пока из рассмотрения и случаи

$$
(m, s)=(k, 2 k),(2 k, k), \quad(m, s)=(k, k),(2 k, 2 k),
$$

в которых расчет асимптотики собственных значений в одномодовой форме (2.3) заведомо невозможен. Связано это с тем, что в первом из них $\lambda_{k, 2 k}^{ \pm}=\lambda_{2 k, k}^{ \pm}=0$, а во-втором $\lambda_{k, k}^{ \pm}=\lambda_{2 k, 2 k}^{\mp}$, т. е. наблюдается резонансное взаимодействие мод. Тем самым, остается разобраться с ситуацией, когда в $(2.3),(2.4)$ индексы $m, s$ таковы, что одновременно

$$
m \neq k, 2 k, \quad s \neq k, 2 k .
$$


При условиях $(2.20)$ для $\mu_{m, s}$ получаем равенство

$$
\mu_{m, s}=-\frac{3 d}{13}
$$

Далее, после определения $\mu_{m, s}$ неоднородность в (2.4) представляет собой линейную комбинацию гармоник (2.7), где случаю $j=1$ соответствуют наборы индекcOB

$$
\begin{aligned}
(r, q)= & (m, s \pm 4 k),(m \pm 2 k, s),(m \pm 2 k, s+4 k),(m \pm 2 k, s-4 k), \\
& (m, s \pm 2 k),(m \pm 4 k, s),(m+4 k, s \pm 2 k),(m-4 k, s \pm 2 k) .
\end{aligned}
$$

В случае же $j=2$ к указанным значениям $(r, q)$ добавляются еще пары

$$
\begin{gathered}
(r, q)=(m+k, s \pm k),(m+k, s \pm 3 k),(m-k, s \pm k),(m-k, s \pm 3 k) \\
(m+3 k, s \pm k),(m+3 k, s \pm 3 k),(m-3 k, s \pm k),(m-3 k, s \pm 3 k),(m, s)
\end{gathered}
$$

Остановимся сначала на проверке условий нерезонансности

$$
\omega_{m, s} \neq \omega_{r, q}
$$

отвечающих случаю $j=1$. Несложным подсчетом находим, что нарушаться неравенства (2.22) могут только при

$$
\begin{aligned}
(r, q)= & (m-2 k, s+4 k),(m+2 k, s-4 k),(m-2 k, s-4 k), \\
& (m+4 k, s-2 k),(m-4 k, s+2 k),(m-4 k, s-2 k),
\end{aligned}
$$

причем соответствуюшие им строгие равенства реализуются при

$$
\begin{aligned}
m & =2 s+5 k, \quad m & =2 s-5 k, \quad m & =5 k-2 s, \\
s & =5 k+2 m, \quad s & =2 m-5 k, \quad s & =5 k-2 m .
\end{aligned}
$$

Рассмотрим группу условий нерезонансности

$$
2 \omega_{k, 2 k} \neq \omega_{m, s}+\omega_{r, q}
$$

соответствующую случаю $j=2$. Как и в предыдущем случае, часть этих условий выполняется автоматически. Нарушаться же могут условия (2.25) при

$$
\begin{gathered}
(r, q)=(m, s-4 k),(m \pm 2 k, s),(m, s \pm 2 k),(m-4 k, s) \\
(m, s),(m+k, s \pm k),(m+k, s-3 k),(m-k, s \pm k) \\
(m-k, s \pm 3 k),(m+3 k, s-k),(m-3 k, s \pm k) \\
(m-3 k, s-3 k)
\end{gathered}
$$


которые эквивалентны соответственно неравенствам

$$
\begin{gathered}
m^{2}+(s-2 k)^{2} \neq k^{2}, \quad s^{2}+(m \pm k)^{2} \neq 4 k^{2}, \quad m^{2}+(s \pm k)^{2} \neq 4 k^{2}, \\
(m-2 k)^{2}+s^{2} \neq k^{2}, \quad m^{2}+s^{2} \neq 5 k^{2}, \quad \Phi_{\mp, \pm}(s)+\Phi_{-,+}(m) \neq 0, \\
\Phi_{-,-}(s)+\Phi_{-,+}(m) \neq 0, \quad \Phi_{+,-}(m)+\Phi_{\mp, \pm}(s) \neq 0, \\
\Phi_{+,-}(m)+\Phi_{ \pm, \pm}(s) \neq 0, \quad \Phi_{+,+}(m)+\Phi_{+,-}(s) \neq 0, \\
\Phi_{-,-}(m)+\Phi_{\mp, \pm}(s) \neq 0, \quad \Phi_{-,-}(m)+\Phi_{-,-}(s) \neq 0,
\end{gathered}
$$

где

$$
\Phi_{ \pm,+}(r)=(r \pm k)(r+2 k), \quad \Phi_{ \pm,-}(r)=(r \pm k)(r-2 k) .
$$

Подводя итог, отметим, что в случае (2.20) функция $z_{m, s}$ может быть определена из краевой задачи (2.4) в требуемом виде только при выполнении условий $(2.22),(2.23)$ и (2.27). Если при некоторых $m$ и $s$ хотя бы одно из этих условий нарушается, то соответствуюшая краевая задача (2.4) оказывается неразрешимой. В связи с этим и другими отмеченными выше исключительными случаями возникает необходимость в подходящей модификации алгоритма, которая предлагается в следуюшем пункте.

2.2. Исследование устойчивости в случае резонансов. Начнем с рассмотрения первого из случаев (2.19), т. е. рассчитаем асимптотику четырех близких к нулю собственных значений краевой задачи (2.1), которым при $\varepsilon=0$ соответствуют собственные функции (1.33) (при $n=2 k$ ). С этой целью введем в рассмотрение матричную краевую задачу

$$
\Pi_{k} V+\varepsilon A_{k}(x, y) V=V \Lambda,\left.\quad V\right|_{x=0, x=1}=\left.V\right|_{y=0, y=1}=0,
$$

где

$$
\begin{gathered}
V=V_{0}(x, y)+\varepsilon V_{1}(x, y), \quad V_{j}=\left[w_{j_{1}}, w_{j_{2}}, w_{j_{3}}, w_{j_{4}}\right], \quad j=0,1, \\
\Lambda=\varepsilon \Lambda_{0}, \quad w_{01}=a_{1} e_{2 k, k}(x, y), \quad w_{02}=a_{2} e_{2 k, k}(x, y), \\
w_{03}=a_{1} e_{k, 2 k}(x, y), \quad w_{04}=a_{2} e_{k, 2 k}(x, y),
\end{gathered}
$$

а столбцы матрицы $V_{1}$, являющиеся двумерными векторами, и элементы постоянной четырехмерной квадратной матрицы $\Lambda_{0}$ подлежат определению. Действуя, далее, обычным образом, т. е. приравнивая в (2.28) коэффициенты при $\varepsilon$, для нахождения $w_{1 j}, j=1, \ldots, 4$, получаем линейные неоднородные краевые задачи

$$
\Pi_{k} w_{1 j}=\sum_{r=1}^{4} \lambda_{r j} w_{0 r}-A_{k}(x, y) w_{0 j},\left.\quad w_{1 j}\right|_{x=0, x=1}=\left.w_{1 j}\right|_{y=0, y=1}=0
$$

а элементы $\lambda_{r j}$ матрищы $\Lambda_{0}$ определяем из условий их разрешимости. В итоге убеждаемся, что $\Lambda_{0}=B$, где $B$ - матрица (1.25).

Напомним, что одно собственное значение матрицы $\Lambda_{0}$ нулевое (что естественно, так как (2.1) - линеаризация на цикле), а три других имеют отрицательные действительные части. Этим, в частности, и объясняется выбор набора (1.19) в качестве решения системы (1.18). В случае же $\alpha_{1}=-c_{0}, \quad \xi_{1}^{0}=\xi_{2}^{0}=2 / \sqrt{21}$ утверждения теорем 1.1, 1.2 остаются в силе, но получаюшиеся при этом циклы 
заведомо неустойчивы: соответствующая им матрица $\Lambda_{0}$ имеет собственное значение в полуплоскости $\{\lambda: \operatorname{Re} \lambda>0\}$.

Случаи $(m, s)=(k, k),(2 k, 2 k)$ (см. (2.19)) также приходится рассматривать совместно, поскольку здесь в силу равенства $\lambda_{k, k}^{+}=\lambda_{2 k, 2 k}^{-}$наблюдается резонансное взаимодействие мод $a_{1} e_{k, k}(x, y), a_{2} e_{2 k, 2 k}(x, y)$. Поэтому в данной ситуации рассмотрению подлежит матричная краевая задача $(2.28)$, в которой

$$
\begin{gathered}
V=V_{0}(x, y)+\varepsilon V_{1}(x, y), \quad V_{j}=\left[w_{j 1}, w_{j 2}\right], \quad j=0,1, \\
w_{01}=a_{1} e_{k, k}(x, y), \quad w_{02}=a_{2} e_{2 k, 2 k}(x, y), \\
\Lambda=\lambda_{k, k}^{+} I+\varepsilon D, \quad D=\left\|d_{j p}\right\|, \quad j, p=1,2 .
\end{gathered}
$$

Здесь $I$ - единичная матрица, а вектор-функции $w_{11}, w_{12}$, являющиеся тригонометрическими полиномами переменных $x, y$, и элементы $d_{j p}$ постоянной матрищы $D$ подлежат определению. Приравнивая затем в (2.28) коэффициенты при $\varepsilon$, для нахождения $w_{1 j}, j=1,2$, приходим к краевым задачам

$$
\begin{gathered}
\left(\Pi_{k}-\lambda_{k, k}^{+} I\right) w_{1 j}=d_{1 j} w_{01}+d_{2 j} w_{02}-A_{k}(x, y) w_{0 j} \\
\left.w_{1 j}\right|_{x=0, x=1}=\left.w_{1 j}\right|_{y=0, y=1}=0
\end{gathered}
$$

анализ которых стандартен. Сначала, приравнивая нулю коэффициенты при $w_{01}$, $w_{02}$ в правых частях, находим

$$
D=\frac{1}{13}\left(\begin{array}{rr}
-11 d & -8 i d \\
8 i \bar{d} & -11 \bar{d}
\end{array}\right)
$$

После этого, как нетрудно убедиться, функции $w_{1 j}$ определяются из (2.32) уже однозначно в том же виде, что и соответствуюшие неоднородности.

Совместному рассмотрению подлежат и случаи $(m, s)=(k, 3 k),(3 k, k)$ (см. $(2.5),(2.11),(2.12))$, поскольку в каждом из них взаимодействуют одни и те же моды $a_{1} e_{k, 3 k}(x, y)$ и $a_{1} e_{3 k, k}(x, y)$. Таким образом, здесь объектом анализа является краевая задача (2.28), в которой сохраняется равенство (2.30), а вместо (2.31) следует положить

$$
\begin{gathered}
w_{01}=a_{1} e_{k, 3 k}(x, y), \quad w_{02}=a_{1} e_{3 k, k}(x, y), \\
\Lambda=\lambda_{k, 3 k}^{+} I+\varepsilon D, \quad D=\left\|d_{j p}\right\|, \quad j, p=1,2 .
\end{gathered}
$$

Действуя далее описанным выше образом, для фигурирующей в (2.34) матрицы $D$ приходим к равенству

$$
D=-\frac{d}{13}\left(\begin{array}{rr}
7 & -4 \\
-4 & 7
\end{array}\right) \text {. }
$$

Несколько сложнее случай, когда в $(2.5),(2.12) p=7 k$. Связано это с тем, что здесь взаимодействуют сразу три моды $a_{1} e_{k, 7 k}(x, y), a_{1} e_{7 k, k}(x, y)$ и $a_{1} e_{5 k, 5 k}(x, y)$, 
а значит, при реализации алгоритма расчета спектра задачи (2.1) на данных модах следует положить в (2.28)

$$
\begin{gathered}
V=V_{0}(x, y)+\varepsilon V_{1}(x, y), \quad V_{j}=\left[w_{j 1}, w_{j 2}, w_{j 3}\right], \quad j=0,1, \\
w_{01}=a_{1} e_{k, 7 k}(x, y), \quad w_{02}=a_{1} e_{7 k, k}(x, y), \quad w_{03}=a_{1} e_{5 k, 5 k}(x, y), \\
\Lambda=\lambda_{k, 7 k}^{+} I+\varepsilon D, \quad D=\left\|d_{j p}\right\|, \quad j, p=1,2,3 .
\end{gathered}
$$

Приравнивая затем коэффициенты при $\varepsilon$, для $w_{1 j}, j=1,2,3$, получим аналогичные (2.32) линейные неоднородные краевые задачи, а элементы неизвестной матрицы $D$ из (2.37) находим из условий их разрешимости. Опуская стандартные вычисления, приведем сразу окончательный результат:

$$
D=-\frac{d}{13}\left(\begin{array}{lll}
7 & 0 & 2 \\
0 & 7 & 2 \\
2 & 2 & 3
\end{array}\right)
$$

Случаи, когда в (2.13) нарушается одно из дополнительных условий $(2.18)$, однотипны. Поэтому изучим, к примеру, резонансное взаимодействие мод

$$
a_{1} e_{2 k, 9 k}(x, y), \quad a_{1} e_{6 k, 7 k}(x, y)
$$

С этой целью положим в $(2.28),(2.30)$

$$
\begin{aligned}
& w_{01}=a_{1} e_{2 k, 9 k}(x, y), \quad w_{02}=a_{1} e_{6 k, 7 k}(x, y), \\
& \Lambda=\lambda_{2 k, 9 k}^{+} I+\varepsilon D, \quad D=\left\|d_{j p}\right\|, \quad j, p=1,2 .
\end{aligned}
$$

В итоге после соответствующих вычислений для матрицы $D$ из (2.39) приходим к равенству

$$
D=-\frac{d}{13}\left(\begin{array}{ll}
7 & 2 \\
2 & 3
\end{array}\right) \text {. }
$$

Отметим также, что матрица (2.40) получается и при резонансном взаимодействии мод $a_{1} e_{9 k, 2 k}(x, y), a_{1} e_{7 k, 6 k}(x, y)$. В других же двух исключительных случаях из (2.13), когда взаимодействуют соответственно пары мод $a_{1} e_{2 k, 7 k / 2}(x, y)$, $a_{1} e_{4 k, k / 2}(x, y)$ и $a_{1} e_{7 k / 2,2 k}(x, y), a_{1} e_{k / 2,4 k}(x, y)$, получается одна и та же матрица

$$
D=-\frac{d}{13}\left(\begin{array}{rr}
7 & -2 \\
-2 & 3
\end{array}\right)
$$

Оставшиеся резонансные случаи связаны с индексами $m, s$ из множества (2.20). Возникающие здесь ситуации весьма разнообразны. Поэтому начнем с изучения двух простейших из них. Предположим сначала, что индексы $m, s$ связаны равенством $m=2 s+5 k$ (см. $(2.24))$, т. е. наблюдается резонансное взаимодействие мод $a_{1} e_{m, s}(x, y)$ и $a_{1} e_{m_{1}, s_{1}}(x, y)$, где $m_{1}=m-2 k, s_{1}=s+4 k$. Тогда, как нетрудно проверить, каждая пара индексов $(m, s)$ и $\left(m_{1}, s_{1}\right)$ удовлетворяет только одному из равенств (2.24) (в частности, $m_{1}=2 s_{1}-5 k$ ) и для обеих пар выполняются 
все условия (2.27). А это означает, что расчет асимптотики собственных значений задачи (2.1) на этих модах следует проводить в форме (2.28), (2.30), где

$$
\begin{gathered}
w_{01}=a_{1} e_{m, s}(x, y), \quad w_{02}=a_{1} e_{m_{1}, s_{1}}(x, y) \\
\Lambda=\lambda_{m, s}^{+} I+\varepsilon D, \quad D=\left\|d_{j p}\right\|, \quad j, p=1,2
\end{gathered}
$$

С помощью несложных вычислений для матрицы $D$ из (2.42) получаем равенство

$$
D=-\frac{d}{13}\left(\begin{array}{ll}
3 & 2 \\
2 & 3
\end{array}\right) \text {. }
$$

Все сказанное выше в полной мере сохраняется и для случая, когда индексы $m$, $s$ связаны соотношением $s=2 m+5 k$. Поэтому здесь реализацию алгоритма также проводим в форме $(2.28),(2.30),(2.42)$, где $m_{1}=m+4 k, s_{1}=s-2 k$, причем для матрищы $D$ получаем прежнее равенство (2.43).

Немного сложнее случай

$$
(m, s): \quad m=2 s-5 k>0, \quad|s-4 k| \neq 0, k, 2 k,
$$

в котором взаимодействуют моды $a_{1} e_{m, s}(x, y)$ и $a_{1} e_{m_{1}, s_{1}}(x, y)$, где $m_{1}=m+2 k$, $s_{1}=|s-4 k|$. Заметим, что равенства $s_{1}=k, 2 k$ в (2.44) исключены из рассмотрения в силу того, что они приводят к уже изученным ситуациям (см. (2.36), (2.37), $(2.39))$. Случай $s_{1}=0$ не имеет смысла по той причине, что $a_{1} e_{m_{1}, 0}(x, y) \equiv 0$.

Фиксируем произвольно индексы $m, s$ из множества (2.44), (2.20). Непосредственной проверкой убеждаемся в том, что каждая пара $(m, s)$ и $\left(m_{1}, s_{1}\right)$ удовлетворяет только одному из равенств $(2.24)$ (в случае $\left(m_{1}, s_{1}\right)$ имеем $m_{1}=2 s_{1}+5 k$ при $s>4 k$ или $m_{1}=5 k-2 s_{1}$ при $s<4 k$ ). При проверке для этих пар выполнения условий (2.27) возникают две исключительные ситуации, которые будут рассмотрены отдельно. А именно, для наборов индексов

$$
\begin{gathered}
(m, s)=(3 k / 5,14 k / 5), \quad\left(m_{1}, s_{1}\right)=(13 k / 5,6 k / 5), \\
\left(m_{2}, s_{2}\right)=(3 k / 5,6 k / 5),
\end{gathered}
$$

а также для наборов

$$
\begin{gathered}
(m, s)=(k / 5,13 k / 5), \quad\left(m_{1}, s_{1}\right)=(11 k / 5,7 k / 5) \\
\left(m_{2}, s_{2}\right)=(4 k / 5,8 k / 5)
\end{gathered}
$$

справедливы равенства вида

$$
\omega_{m, s}=\omega_{m_{1}, s_{1}}, \quad 2 \omega_{k, 2 k}=\omega_{m, s}+\omega_{m_{2}, s_{2}}, \quad 2 \omega_{k, 2 k}=\omega_{m_{1}, s_{1}}+\omega_{m_{2}, s_{2}} .
$$

Все остальные условия нерезонансности (2.22), (2.25) для данных наборов индексов выполняются.

Итак, пусть не имеет места ни один из исключительных случаев (2.45), (2.46). Тогда реализация алгоритма для данных пар индексов $(m, s)$ и $\left(m_{1}, s_{1}\right)$ проводится в прежнем виде $(2.28),(2.30),(2.42)$, а для матрицы $D$ получается формула

$$
D=-\frac{d}{13}\left(\begin{array}{rr}
3 & \pm 2 \\
\pm 2 & 3
\end{array}\right)
$$


где знак + соответствует неравенству $s>4 k$, а знак - возникает при $s<4 k$.

Случай $(m, s): s=2 m-5 k>0,|m-4 k| \neq 0, k, 2 k$, не нуждается в отдельном рассмотрении, так как фактически сводится к предыдушему посредством замен $m \rightarrow s, s \rightarrow m$. В частности, здесь также возникают две исключительные ситуащии:

$$
\begin{gathered}
(m, s)=(14 k / 5,3 k / 5), \quad\left(m_{1}, s_{1}\right)=(6 k / 5,13 k / 5) \\
\left(m_{2}, s_{2}\right)=(6 k / 5,3 k / 5) \\
(m, s)=(13 k / 5, k / 5), \quad\left(m_{1}, s_{1}\right)=(7 k / 5,11 k / 5) \\
\left(m_{2}, s_{2}\right)=(8 k / 5,4 k / 5) .
\end{gathered}
$$

В остальных ситуациях применяется прежний алгоритм (2.28), (2.30), (2.42), а для матрицы $D$ сохраняется равенство $(2.48)$, в котором знаку + соответствует условие $m>4 k$, а знак - выбирается при $m<4 k$.

Обратимся теперь к случаям, когда в дополнение к $(2.20)$ имеем $m=5 k-2 s>0$, $|m-2 k| \neq k, 2 k ; 4 k-s \neq 0, k$ или $s=5 k-2 m>0,|s-2 k| \neq k, 2 k$; $4 k-m \neq 0, k$. Заметим, что если исключить ситуации $(2.45)-(2.47),(2.49),(2.50)$, а также случай резонансного взаимодействия мод

$$
\begin{gathered}
w_{01}=a_{1} e_{5 k / 3,5 k / 3}(x, y), \quad w_{02}=a_{1} e_{k / 3,7 k / 3}(x, y), \\
w_{03}=a_{1} e_{7 k / 3, k / 3}(x, y)
\end{gathered}
$$

то и здесь алгоритм сохраняет прежнюю форму $(2.28),(2.30),(2.42)$, где $m_{1}=$ $|m-2 k|, s_{1}=4 k-s$ или $m_{1}=4 k-m, s_{1}=|s-2 k|$ соответственно. Для матрищы $D$ оказывается справедливым равенство (2.48), в котором в первом случае знаки + или - выбираются в соответствии с условиями $m<2 k$ или $m>2 k$, а во втором случае-в соответствии с условиями $s<2 k$ или $s>2 k$.

Анализ четырех обнаруженных выше исключительных случаев (2.45), (2.46), $(2.49),(2.50)$ производится схожим образом, так как в силу равенств $(2.47)$ здесь взаимодействуют моды

$$
w_{01}=a_{1} e_{m, s}(x, y), \quad w_{02}=a_{1} e_{m_{1}, s_{1}}(x, y), \quad w_{03}=a_{2} e_{m_{2}, s_{2}}(x, y),
$$

где пары индексов $(m, s),\left(m_{1}, s_{1}\right),\left(m_{2}, s_{2}\right)$ принимают соответственно значения $(2.45),(2.46),(2.49),(2.50)$. Поэтому для расчета асимптотики собственных значений краевой задачи (2.1) на данных модах подставим в (2.28) равенства $(2.36),(2.37)$, в которых теперь $w_{0 j}, j=1,2,3$, задаются формулами $(2.52)$, a $\lambda_{k, 7 k}^{+}$заменено на $\lambda_{m, s}^{+}$. Действуя далее обычным образом, убеждаемся, что в случаях $(2.45),(2.49)$ матрица $D$ определяется соответственно равенствами

$$
D=\frac{1}{13}\left(\begin{array}{ccc}
-3 d & 2 d & \pm 2 d \\
2 d & -3 d & \mp 2 d \\
\pm 2 \bar{d} & \mp 2 \bar{d} & -3 \bar{d}
\end{array}\right)
$$

а в случаях (2.46), (2.50) - одним и тем же равенством

$$
D=\frac{1}{13}\left(\begin{array}{ccc}
-3 d & 2 d & 2 i d \\
2 d & -3 d & -2 i d \\
-2 i \bar{d} & 2 i \bar{d} & -3 \bar{d}
\end{array}\right)
$$


Исследование оставшегося из пяти исключительных случаев, а именно резонансного взаимодействия мод (2.51), проводится по схеме $(2.36),(2.37)$, где, естественно, $\lambda_{k, 7 k}^{+}$следует заменить на $\lambda_{5 k / 3,5 k / 3}^{+}$, а функции $w_{0 j}, j=1,2,3$, определить формулами (2.51). Не останавливаясь на вполне понятных вычислениях, приведем сразу окончательный результат:

$$
D=-\frac{d}{13}\left(\begin{array}{lll}
3 & 2 & 2 \\
2 & 3 & 0 \\
2 & 0 & 3
\end{array}\right)
$$

Заключительный этап расчета асимптотики собственных значений задачи (2.1) состоит в рассмотрении тех резонансных случаев, когда для индексов $(m, s)$ из множества (2.20) выполняются условия (2.22), (2.23), но нарушается по крайней мере одно из неравенств (2.27). Анализ этих неравенств показывает, что помимо двух особых случаев (о которых скажем чуть позже) все возникающие здесь ситуации укладываются в следуюшую схему: пары индексов $(m, s)$ и $\left(m_{1}, s_{1}\right)=(r, q)$, где $(r, q)$ - одно из значений $(2.26)$, связаны соотношением

$$
2 \omega_{k, 2 k}=\omega_{m, s}+\omega_{m_{1}, s_{1}},
$$

а все остальные условия нерезонансности (2.22), (2.25) для каждой из этих пар выполняются. Иными словами, резонансно взаимодействуют только моды

$$
w_{01}=a_{1} e_{m, s}(x, y), \quad w_{02}=a_{2} e_{m_{1}, s_{1}}(x, y) .
$$

В этом случае, как и в других рассмотренных выше аналогичных ситуациях, реализация алгоритма проводится в форме (2.28), (2.30), (2.42), где, естественно, функции $w_{01}, w_{02}$ определены равенствами (2.56). Результатом алгоритма здесь являются двумерные матрицы одного из следующих видов:

$$
D=\left(\begin{array}{cc}
-\frac{3}{13} d & \pm \frac{2}{13} d \\
\pm \frac{2}{13} \bar{d} & -\frac{3}{13} \bar{d}
\end{array}\right), \quad\left(\begin{array}{cc}
-\frac{3}{13} d & 0 \\
0 & -\frac{3}{13} \bar{d}
\end{array}\right), \quad\left(\begin{array}{cc}
-\frac{3}{13} d & \mp \frac{2 i}{13} d \\
\pm \frac{2 i}{13} \bar{d} & -\frac{3}{13} \bar{d}
\end{array}\right)
$$

Итак, для завершения алгоритмической части доказательства теоремы 1.3 осталось разобраться только с двумя упомянутыми вьше исключительными случаями. Первый из них связан с набором индексов

$$
\begin{gathered}
\left(m_{0}, s_{0}\right)=(4 k / 5,13 k / 5), \quad\left(m_{1}, s_{1}\right)=(k / 5,8 k / 5), \\
\left(m_{2}, s_{2}\right)=(4 k / 5,7 k / 5), \quad\left(m_{3}, s_{3}\right)=(11 k / 5,8 k / 5),
\end{gathered}
$$

а второй - с набором, получающимся из (2.58) при заменах $\left(m_{j}, s_{j}\right) \rightarrow\left(s_{j}, m_{j}\right)$, $j=0, \ldots, 3$. Особенность данных случаев состоит в том, что здесь в силу равенств

$$
2 \omega_{k, 2 k}=\omega_{m_{0}, s_{0}}+\omega_{m_{j}, s_{j}}, \quad 2 \omega_{k, 2 k}=\omega_{m_{3}, s_{3}}+\omega_{m_{j}, s_{j}}, \quad j=1,2,
$$

резонансно взаимодействуют сразу четыре моды:

$$
\begin{array}{ll}
w_{01}=a_{1} e_{m_{0}, s_{0}}(x, y), & w_{02}=a_{2} e_{m_{1}, s_{1}}(x, y), \\
w_{03}=a_{2} e_{m_{2}, s_{2}}(x, y), & w_{04}=a_{1} e_{m_{3}, s_{3}}(x, y) .
\end{array}
$$

Поэтому при реализации алгоритма на указанных модах подставим в (2.28) матрицы

$$
\begin{gathered}
V=V_{0}(x, y)+\varepsilon V_{1}(x, y), \quad V_{j}=\left[w_{j 1}, w_{j 2}, w_{j 3}, w_{j 4}\right], \quad j=0,1, \\
\Lambda=\lambda_{m_{0}, s_{0}}^{+} I+\varepsilon D, \quad D=\left\|d_{j p}\right\|, \quad j, p=1, \ldots, 4
\end{gathered}
$$


В итоге убеждаемся, что в первом и втором случае матрица $D$ задается соответственно равенствами

$$
D=\frac{1}{13}\left(\begin{array}{cccc}
-3 d & 2 i d & \pm 2 d & 0 \\
-2 i \bar{d} & -3 \bar{d} & 0 & \mp 2 \bar{d} \\
\pm 2 \bar{d} & 0 & -3 \bar{d} & 2 i \bar{d} \\
0 & \mp 2 d & -2 i d & -3 d
\end{array}\right)
$$

2.3. Завершение доказательства теоремы 1.3. Проблема обоснования теоремы 1.3 сводится, очевидно, к обоснованию описанного вьше алгоритма расчета асимптотики собственных значений задачи (2.1). Соответствующий анализ разобьем на два этапа. На первом этапе введем в рассмотрение вектор-функции

$$
v_{m, s}^{j}(x, y, \varepsilon)=v_{m, s, 0}^{j}(x, y)+\varepsilon v_{m, s, 1}^{j}(x, y), \quad j=1, \ldots, j_{m, s}^{*},
$$

которые определяются следуюшим образом. В случае, когда для пары индексов $(m, s)$ алгоритм реализуется в одномодовом виде $(2.3)$, полагаем в $(2.60)$

$$
j_{m, s}^{*}=1, \quad v_{m, s}^{1}=a_{1} e_{m, s}(x, y)+\varepsilon z_{m, s}(x, y),
$$

где $z_{m, s}$ - решение задачи (2.4). Далее, если при $(m, s) \neq(2 k, k),(k, 2 k)$ имеем резонансньй случай, то $j_{m, s}^{*}$ считаем равным количеству взаимодействующих мод, а в качестве функций (2.60) берем столбцы соответствующей матрицы $V_{0}+\varepsilon V_{1}$ (см. $(2.30),(2.36)$ и т. д.). И наконец, при $(m, s)=(2 k, k),(k, 2 k)$ положим

$$
j_{2 k, k}^{*}=j_{k, 2 k}^{*}=1, \quad v_{2 k, k}^{1}=w_{01}+\varepsilon w_{11}, \quad v_{k, 2 k}^{1}=w_{03}+\varepsilon w_{13},
$$

где $w_{j 1}, w_{j 3}, j=0,1,-$ первый и третий столбцы матриц $V_{0}, V_{1}$ из $(2.29)$.

На втором этапе выполним в краевой задаче (1.44) замену переменных

$$
z=\sum_{m, s=1}^{\infty} \sum_{j=1}^{j_{m, s}^{*}}\left(\xi_{m, s}^{j} v_{m, s}^{j}(x, y, \varepsilon)+\eta_{m, s}^{j}\left[v_{m, s}^{j}(x, y, \varepsilon)\right]^{*}\right)
$$

где, как и в равенстве $(1.31)$, операция $*$ означает, что $v^{*}=\operatorname{colon}\left(\bar{v}_{2}, \bar{v}_{1}\right)$, если $v=$ colon $\left(v_{1}, v_{2}\right)$, а относительно комплексных постоянных $\xi_{m, s}^{j}, \eta_{m, s}^{j}$ предполагаем, что $\xi=\left\{\xi_{m, s}^{j}, \eta_{m, s}^{j}: m, s \geqslant 1\right\} \in l_{2}$, т.е. сходится ряд

$$
\sum_{m, s=1}^{\infty} \sum_{j=1}^{j_{m, s}^{*}}\left(\left|\xi_{m, s}^{j}\right|^{2}+\left|\eta_{m, s}^{j}\right|^{2}\right)
$$

Основная трудность, с которой обычно сталкиваемся при работе с бесконечномерными заменами, состоит в решении так называемой проблемы малых знаменателей. Однако в нашем случае данная проблема не возникает. Связано это с тем, что каждая функция $v_{m, s, 1}^{j}(x, y)$ из $(2.60)$ представляет собой линейную комбинацию конечного (не зависящего от $m, s, k$ ) числа гармоник (2.7) с коэффициентами вида

$$
\text { const } \cdot\left(\omega_{m, s}-\omega_{r, q}\right)^{-1}, \quad \text { const } \cdot\left(2 \omega_{k, 2 k}-\omega_{m, s}-\omega_{r, q}\right)^{-1},
$$


причем для соответствующих знаменателей заведомо справедливы оценки

$$
\left|\omega_{m, s}-\omega_{r, q}\right| \geqslant \pi^{2}, \quad\left|2 \omega_{k, 2 k}-\omega_{m, s}-\omega_{r, q}\right| \geqslant \pi^{2}
$$

Отсюда и из того факта, что при $\varepsilon=0$ вектор-функции (2.60) образуют ортонормированный базис в пространстве $E=L_{2}(\Omega) \times L_{2}(\Omega)$, следует, что равенство $(2.61)$ индуцирует линейный оператор из $l_{2}$ в $E$, ограниченный равномерно по $\varepsilon$ и $k \geqslant 1$ вместе со своим обратным. Иными словами, замена (2.61) является равномерно корректной.

Из способа построения функций (2.60) вытекает также, что результатом проведения замены (2.61) в краевой задаче (1.44) оказывается спектральная задача в $l_{2}$ :

$$
\left(L_{1, k}(\varepsilon)+\varepsilon^{2} L_{2, k}(\varepsilon)\right) \xi=\lambda \xi
$$

где линейный оператор $L_{2, k}(\varepsilon)$ ограничен равномерно по $k \geqslant 1$ и $\varepsilon$, а $L_{1, k}(\varepsilon)$ имеет блочно-диагональную структуру вида

$$
L_{1, k}(\varepsilon) \xi=\left\{\widetilde{\xi}_{m, s}^{j}, \widetilde{\eta}_{m, s}^{j}: j=1, \ldots, j_{m, s}^{*}, m, s \geqslant 1\right\}
$$

Здесь в нерезонансных случаях компоненты $\widetilde{\xi}_{m, s}^{1}, \widetilde{\eta}_{m, s}^{1}$ задаются равенствами

$$
\widetilde{\xi}_{m, s}^{1}=\left(\lambda_{m, s}^{+}+\varepsilon \mu_{m, s}\right) \xi_{m, s}^{1}, \quad \widetilde{\eta}_{m, s}^{1}=\left(\lambda_{m, s}^{-}+\varepsilon \bar{\mu}_{m, s}\right) \eta_{m, s}^{1}
$$

Далее, в случае резонансного набора $(m, s) \neq(k, 2 k),(2 k, k)$ в качестве $\widetilde{\xi}_{m, s}^{j}, \widetilde{\eta}_{m, s}^{j}$ берутся соответственно координаты векторов

$$
\left(\lambda_{m, s}^{+} I+\varepsilon D\right) \xi_{m, s}, \quad\left(\lambda_{m, s}^{-} I+\varepsilon \bar{D}\right) \eta_{m, s}
$$

где $D$ - матрища из алгоритма, отвечающая данному резонансному случаю, а $\xi_{m, s}$ и $\eta_{m, s}$ - векторы с компонентами $\xi_{m, s}^{j}$ и $\eta_{m, s}^{j}, j=1, \ldots, j_{m, s}^{*}$. Наконец, при $(m, s)=(k, 2 k),(2 k, k)$ справедливо соотношение

$$
\operatorname{colon}\left(\widetilde{\xi}_{2 k, k}^{1}, \widetilde{\eta}_{2 k, k}^{1}, \widetilde{\xi}_{k, 2 k}^{1}, \widetilde{\eta}_{k, 2 k}^{1}\right)=\varepsilon B v
$$

где $B$ - матрица из $(1.25), v=\operatorname{colon}\left(\xi_{2 k, k}^{1}, \eta_{2 k, k}^{1}, \xi_{k, 2 k}^{1}, \eta_{k, 2 k}^{1}\right)$.

Из структуры оператора (2.63) следует, что его спектр-это числа $\lambda_{m, s}^{+}+\varepsilon \mu_{m, s}$, $\lambda_{m, s}^{-}+\varepsilon \bar{\mu}_{m, s}$ (см. (2.64)) и совокупность собственных значений матриц из (2.65), (2.66). Заметим, далее, что в силу равенств $(2.6),(2.14),(2.21)$ все фигурирующие в $(2.64)$ постоянные $\mu_{m, s}$ имеют отрицательные действительные части. Опираясь 
на явньй вид (2.33), (2.35), (2.38), (2.40), (2.41), (2.43), (2.48), (2.53)-(2.55), (2.57), $(2.59)$ матриц $D$ из (2.65), нетрудно убедиться в том, что и они являются гурвицевыми. Исключение составляет только матрица $B$ из (2.66), имеющая одно простое нулевое собственное значение и три собственных значения в полуплоскости $\{\lambda: \operatorname{Re} \lambda<0\}$. Тем самым, заведомо найдется такая не зависяшая от $\varepsilon$ и $k \geqslant 1$ постоянная $\gamma_{0}>0$, что весь спектр операторов $L_{1, k}(\varepsilon), k \geqslant 1$ (за исключением упомянутых выше простых нулевых собственных значений), содержится в комплексной полуплоскости $\left\{\lambda: \operatorname{Re} \lambda \leqslant-\gamma_{0} \varepsilon\right\}$.

При переходе от операторов $L_{1, k}(\varepsilon)$ к исходным спектральным задачам $(2.62)$ в силу равномерной по $\varepsilon$ и $k \geqslant 1$ ограниченности “добавок" $L_{2, k}(\varepsilon)$ описанная ситуация сохраняется. А именно, найдется такое достаточно малое $\varepsilon_{0}>0$, что при всех $0<\varepsilon \leqslant \varepsilon_{0}$ нулевое собственное значение каждой из этих задач по-прежнему является простым, а остальные их точки спектра находятся в той же самой полуплоскости $\left\{\lambda: \operatorname{Re} \lambda \leqslant-\gamma_{0} \varepsilon\right\}$. Теорема 1.3 полностью доказана.

Следует отметить, что семействами (1.43) исчерпываются все устойчивые автомодельные циклы краевой задачи (1.8), которые удается обнаружить локальными методами. Действительно, при

$$
n \neq k, \quad n \neq 2 k, \quad k \neq 2 n
$$

любой цикл (1.11), доставляемый теоремой 1.1, является неустойчивым (с размерностью неустойчивого многообразия, равной трем). Связано это с тем, что здесь реализация описанного выше алгоритма исследования устойчивости в одном из случаев, а именно при резонансном взаимодействии мод $a_{1} e_{n+k, n-k}(x, y)$ и $a_{1} e_{n-k, n+k}(x, y)$, приводит к матрище

$$
D=-\frac{d}{13}\left(\begin{array}{rr}
3 & -4 \\
-4 & 3
\end{array}\right)
$$

имеющей собственное значение с положительной действительной частью. Далее, если отказаться от требования аналитичности по $\varepsilon$ фигурирующих в (1.11) амплитуды $u_{n, k}$ и частоты $\alpha_{n, k}$, то аналог теоремы 1.1 справедлив без дополнительных предположений (1.13) относительно номеров $n, k$ (при ее обосновании в основном используется прежняя схема, но вместо оператора П следует взять оператор вида (2.1), получающийся при линеаризации на приближенном цикле и имеющий в подходящем подпространстве коразмерности один норму обратного порядка $\left.\varepsilon^{-1}\right)$. Но и в этом случае доставляемые данной теоремой циклы, отличные от (1.43), оказываются неустойчивыми.

В заключение отметим, что все сказанное вьше отнюдь не исключает сушествования у краевой задачи (1.8) наряду с циклами (1.43) устойчивых автоколебательных режимов более сложной природы. В самом деле, характер неустойчивости циклов (1.11), (1.27), (1.28) при условии (2.67) порождает вопрос о сушествовании устойчивых двумерных торов, сосредоточенных на модах $e_{n, k}, e_{k, n}, e_{n+k, n-k}$, $e_{n-k, n+k}$. Однако разрешение этого вопроса сопряжено со значительными аналитическими трудностями и требует отдельного рассмотрения, выходящего за рамки настоящей статьи. 


\section{§3. Уравнение Шрёдингера с граничными условиями Неймана}

3.1. Исследование гипербуферности в прямоугольнике и квадрате. Рассмотрим случай прямоугольника с неравными сторонами, т. е. в области

$$
\Omega=\{(x, y): 0 \leqslant x \leqslant 1,0 \leqslant y \leqslant l\}
$$

рассмотрим краевую задачу

$$
u_{t}+i \Delta u=\varepsilon\left(u-d|u|^{2} u\right),\left.\quad u_{x}\right|_{x=0, x=1}=\left.u_{y}\right|_{y=0, y=l}=0
$$

Заметим, что эта задача допускает, очевидно, решения, зависящие только от одной пространственной переменной $x$ или $y$. В частности, из результатов монографии [1] следует существование такого достаточно малого числа $\varepsilon_{0}>0$, что при всех $0<\varepsilon \leqslant \varepsilon_{0}$ задача (3.2) имеет счетное число автомодельных циклов

$$
u=u_{n}^{1}(x, \varepsilon) \exp \left(i \omega_{n}^{1}(\varepsilon) t\right), \quad u=u_{n}^{2}(y, \varepsilon) \exp \left(i \omega_{n}^{2}(\varepsilon) t\right), \quad n \geqslant 1
$$

Здесь достаточно гладкие по своим переменным функции $u_{n}^{j}, \omega_{n}^{j}, j=1,2$, таковы, что равномерно по $n \geqslant 1, x \in[0,1], y \in[0, l]$

$$
\begin{array}{cc}
u_{n}^{1}(x, \varepsilon)=\frac{2}{\sqrt{3}} \cos (n \pi x)+O(\varepsilon), & u_{n}^{2}(y, \varepsilon)=\frac{2}{\sqrt{3}} \cos \left(\frac{n \pi y}{l}\right)+O(\varepsilon), \\
\omega_{n}^{1}(\varepsilon)=n^{2} \pi^{2}-c_{0} \varepsilon+O\left(\varepsilon^{2}\right), & \omega_{n}^{2}(\varepsilon)=\frac{n^{2} \pi^{2}}{l^{2}}-c_{0} \varepsilon+O\left(\varepsilon^{2}\right) .
\end{array}
$$

Кроме того, в [1] установлена равномерная экспоненщиальная орбитальная устойчивость всех циклов (3.3), (3.4) по отношению к возмушениям начальных условий, зависящим только от $x$ или $y$ соответственно. Тем самым, естественным образом возникает проблема исследования их устойчивости по отношению к произвольным возмущениям начальных условий $(u, \bar{u}) \in \stackrel{\circ}{W_{2}^{2}}(\Omega) \times \stackrel{\circ}{W} \underset{2}{2}(\Omega)$, где $\stackrel{\circ}{W} \underset{2}{2}-$ замыкание в метрике $W_{2}^{2}$ линеала гладких функций, удовлетворяющих нулевым граничным условиям Неймана. Решение этой проблемы приводится ниже.

ТЕОРема 3.1. Фиксируем произвольно натуральное $n_{0}$ и будем считать параметр $l$ из (3.1) таковым, что при каждом $n=1,2, \ldots, n_{0}$ и при всех иельх $k \geqslant 0, \quad m \geqslant 1$ выполняются неравенства

$$
k^{2}+\frac{m^{2}}{l^{2}} \neq n^{2}, \quad k^{2}+m^{2} l^{2} \neq n^{2} .
$$

Тогда найдется такое достаточно малое $\varepsilon_{0}=\varepsilon_{0}\left(n_{0}, l\right)>0$, ито при $0<\varepsilon \leqslant$ $\varepsilon_{0}$ иикль (3.3) краевой задачи (3.2) с номерами $n=1,2, \ldots, n_{0}$ әкспоненииально орбитально устойчивы. 
ДоКАЗАТЕЛЬСТво. Фиксируем произвольно номер $n \leqslant n_{0}$ и покажем устойчивость соответствующего цикла семейства (3.3), зависящего только от $x$ (случай цикла, зависяшего от $y$, разбирается аналогично). Как и при доказательстве теоремы 1.3 , интересующая нас проблема с учетом формул (3.4) сводится к анализу расположения спектра аналогичной (2.1) краевой задачи

$$
\Pi_{n} h+\varepsilon A_{n}(x) h=\lambda h,\left.\quad h_{x}\right|_{x=0, x=1}=\left.h_{y}\right|_{y=0, y=l}=0,
$$

где $h=\operatorname{colon}\left(h_{1}, h_{2}\right)$

$$
\begin{gathered}
\Pi_{n}=\operatorname{diag}\left\{-i\left(\Delta+\pi^{2} n^{2}\right), i\left(\Delta+\pi^{2} n^{2}\right)\right\}, \quad A_{n}(x)=\left(\begin{array}{cc}
\varkappa_{1, n}(x) & \varkappa_{2, n}(x) \\
\varkappa_{2, n}(x) & \varkappa_{1, n}(x)
\end{array}\right), \\
\varkappa_{1, n}=d\left(1-\frac{8}{3} \cos ^{2}(n \pi x)\right), \quad \varkappa_{2, n}=-\frac{4}{3} d \cos ^{2}(n \pi x) .
\end{gathered}
$$

При исследовании этой краевой задачи удобно положить

$$
h=\sum_{m=0}^{\infty} h_{m}(x) \cos \left(\frac{m \pi y}{l}\right) .
$$

В результате в силу независимости ее коэффициентов от $y$ она "расслаивается" на счетное число краевых задач для $h=h_{m}(x)$ :

$$
\Pi_{m, n} h+\varepsilon A_{n}(x) h=\lambda h,\left.\quad \frac{d h}{d x}\right|_{x=0, x=1}=0, \quad m=0,1,2, \ldots,
$$

где

$$
\Pi_{m, n}=\operatorname{diag}\left\{-i L_{m, n}, i L_{m, n}\right\}, \quad L_{m, n}=\frac{d^{2}}{d x^{2}}+\pi^{2} n^{2}-\frac{\pi^{2} m^{2}}{l^{2}} .
$$

Отметим, что случай $m=0$ в (3.7) рассматривать нет необходимости, так как, что уже отмечалось выше, интересуюший нас цикл устойчив по отношению к возмушениям начальных условий, зависяшим только от $x$. В случае же $m \geqslant 1$ при $\varepsilon=0$ спектр каждой из задач (3.7) состоит из чисто мнимых собственных значений

$$
\lambda_{k}, \bar{\lambda}_{k}: \quad \lambda_{k}=i \pi^{2}\left(k^{2}+\frac{m^{2}}{l^{2}}-n^{2}\right), \quad k=0,1, \ldots
$$

которым отвечают собственные функции

$$
v_{k}(x)=a_{1} \cos (k \pi x), \quad w_{k}(x)=a_{2} \cos (k \pi x),
$$

где, напомним, векторы $a_{1}, a_{2}-$ те же, что и в (1.33).

Как и в п. 2.1 , для нахождения поправок порядка $\varepsilon$ к собственным значениям (3.8) подставим в (3.7) выражения

$$
h=v_{k}(x)+\varepsilon v_{k, 1}(x), \quad \lambda=\lambda_{k}+\varepsilon \lambda_{k, 1}, \quad k=0,1, \ldots,
$$


и приравняем коэффищиенты при $\varepsilon$. В итоге приходим к уравнению

$$
\left(\lambda_{k} I-\Pi_{m, n}\right) v_{k, 1}=A_{n}(x) v_{k}(x)-\lambda_{k, 1} v_{k}(x)
$$

решение которого в случае $k \neq n$ ищем в виде линейной комбинации функций $\cos (k \pi x), \cos ((k \pm 2 n) \pi x)$. На этом пути сначала, приравнивая нулю в его правой части коэффищиент при $a_{1} \cos (k \pi x)$, находим

$$
\lambda_{k, 1}=-\frac{d}{3}
$$

а затем определяем и функцию $v_{k, 1}(x)$, имеющую вид

$$
v_{k, 1}(x)=r_{1} \cos (k \pi x)+r_{2} \cos ((k+2 n) \pi x)+r_{3} \cos ((k-2 n) \pi x),
$$

где

$$
\begin{gathered}
r_{1}=\operatorname{colon}\left(r_{1}^{1}, r_{1}^{2}\right), \quad r_{2}=\operatorname{colon}\left(r_{+}^{1}, r_{+}^{2}\right), \quad r_{3}=\operatorname{colon}\left(r_{-}^{1}, r_{-}^{2}\right) \\
r_{1}^{1}=0, \quad r_{1}^{2}=\frac{i \bar{d}}{3 \pi^{2}\left(k^{2}+m^{2} / l^{2}-n^{2}\right)}, \quad r_{ \pm}^{1}=-\frac{i d}{6 \pi^{2} n(n \pm k)} \\
r_{ \pm}^{2}=\frac{i \bar{d}}{6 \pi^{2}\left((n \pm k)^{2}+m^{2} / l^{2}\right)}
\end{gathered}
$$

Отметим, что знаменатели в формулах для $r_{1}^{2}$ отличны от нуля в силу условий теоремы (см. (3.5)).

В случае $k=n$ рассмотрению подлежит то же самое уравнение $(3.10)$, но теперь его решение следует искать в виде линейной комбинации только двух функций $\cos (n \pi x)$ и $\cos (3 n \pi x)$. Проводя соответствующие вычисления, убеждаемся, что здесь

$$
\lambda_{n, 1}=-d, \quad v_{n, 1}=r_{1} \cos (n \pi x)+r_{2} \cos (3 n \pi x),
$$

где $r_{1}=\operatorname{colon}\left(0, i \bar{d} l^{2} /\left(2 \pi^{2} m^{2}\right)\right)$, а для компонент вектора $r_{2}$ справедливы прежние формулы $(3.13)$ (при $k=n)$.

Для обоснования полученных асимптотических формул сделаем при каждом $m \geqslant 1$ в краевой задаче (3.7) замену переменных

$$
h=\sum_{k=0}^{\infty} \eta_{k, 1}\left(v_{k}(x)+\varepsilon v_{k, 1}(x)\right)+\eta_{k, 2}\left(w_{k}(x)+\varepsilon w_{k, 1}(x)\right),
$$

где $\eta=\operatorname{colon}\left(\eta_{0,1}, \eta_{0,2}, \ldots, \eta_{k, 1}, \eta_{k, 2}, \ldots\right) \in l_{2}, w_{k, 1}=v_{k, 1}^{*}$, а операция $*$ та же, что и в (2.61). Из описанного вьше способа построения входящих в правую часть формулы (3.15) функций следует, что, во-первых, равенством (3.15) задается ограниченный (вместе со своим обратным) линейный оператор, действуюший из $l_{2}$ в $L_{2}(0,1) \times L_{2}(0,1)$; во-вторых, результатом выполнения данной замены в $(3.7)$ является спектральная задача в $l_{2}$ :

$$
\left(\Gamma_{0, m, n}+\varepsilon^{2} \Gamma_{1, m, n}(\varepsilon)\right) \eta=\lambda \eta,
$$


где

$$
\Gamma_{0, m, n}=\operatorname{diag}\left\{\lambda_{0}+\varepsilon \lambda_{0,1}, \bar{\lambda}_{0}+\varepsilon \bar{\lambda}_{0,1}, \ldots, \lambda_{k}+\varepsilon \lambda_{k, 1}, \bar{\lambda}_{k}+\varepsilon \bar{\lambda}_{k, 1}, \ldots\right\}
$$

а линейньй оператор $\Gamma_{1, m, n}(\varepsilon)$ ограничен равномерно по $\varepsilon$.

Итак, проблема устойчивости выбранного нами цикла свелась к исследованию расположения спектра оператора $\Gamma_{0, m, n}+\varepsilon^{2} \Gamma_{1, m, n}$ из (3.16). В связи с этим обратим внимание на следующие обстоятельства: в силу (3.8), (3.11), (3.14) все собственные значения диагонального оператора (3.17) имеют отрицательные действительные части порядка $\varepsilon$, а из характера зависимости от $m$ входящих в (3.15) функций $v_{k, 1}, w_{k, 1}$ (см. (3.12)-(3.14)) вытекает, что оператор $\Gamma_{1, m, n}$ равномерно ограничен также и по $m \geqslant 1$. Тем самым, по любому фиксированному $\gamma_{0} \in(0,1 / 3)$ можно указать такое $\varepsilon_{0}=\varepsilon_{0}\left(n_{0}, l, \gamma_{0}\right)>0$, что при всех $0<\varepsilon \leqslant \varepsilon_{0}$ спектр каждой из краевых задач (3.7) при $m \geqslant 1, n=1,2, \ldots, n_{0}$ лежит в комплексной полуплоскости $\left\{\lambda: \operatorname{Re} \lambda \leqslant-\gamma_{0} \varepsilon\right\}$. Теорема доказана.

Отдельно остановимся на случае $l=1$, который в силу условий (3.5) оказьвается исключенным из рассмотрения в рамках теоремы 3.1. При $l=1$, как нетрудно увидеть, циклы (3.3) становятся симметричными, т. е. переходят друг в друга в результате замен $x \rightarrow y, y \rightarrow x$. Обозначим, далее, через $S$ - совокупность таких номеров $n$, что $n^{2} \neq k^{2}+m^{2}$ при любых натуральных $k, m$. Справедливо следующее утверждение.

Tеорема 3.2. Пусть в (3.1) $l=1$. Тогда найдется такое достаточно малое $\varepsilon_{0}>0$, что при всех $0<\varepsilon \leqslant \varepsilon_{0}$ и при каждом $n \in S$ соответствующая пара циклов (3.3) краевой задачи (3.2) әкспоненциально орбитально устойчива.

Для доказательства достаточно заметить, что при любом $n \in S$ все проделанные в процессе обоснования теоремы 3.1 построения сохраняются и, в частности, оператор $\Gamma_{1, m, n}$ из (3.16) оказывается равномерно ограниченным по $\varepsilon, m \geqslant 1$, $n \in S$ (малые знаменатели в (3.13) не появляются, так как при каждом $n \in S$ имеем $\left.\left|k^{2}+m^{2}-n^{2}\right| \geqslant 1\right)$.

Из теоремы 3.2 и того факта, что множество $S$ состоит из бесконечного числа элементов, вытекает, что при $l=1$ в рамках краевой задачи $(3.2)$ реализуется феномен гипербуферности. Более того, как и циклы (1.43) задачи (1.8), соответствующие циклы (3.3) обладают свойством равномерной устойчивости по $n \in S$, т. е. для них справедлив аналог теоремы 1.3. Действительно, существование требуемой постоянной $\gamma_{0}>0$ для краевых задач (3.7) в случае $m \geqslant 1, n \in S$ следует из упомянутых выше свойств оператора $\Gamma_{1, m, n}(\varepsilon)$, а в случае $m=0, n \geqslant 1$ - из результатов монографии [1].

Предположим теперь, что по-прежнему $l=1$, а номер $n$ удовлетворяет равенству $n^{2}=k_{0}^{2}+m_{0}^{2}$ при некоторых натуральных $k_{0}$ и $m_{0}$. Тогда соответствующая пара циклов (3.3) экспоненциально неустойчива. Действительно, в этом случае при $\varepsilon=0$ краевая задача (3.7) с номером $m_{0}$ имеет двукратное нулевое собственное значение, которому отвечают собственные функции $(3.9)$ при $k=k_{0}$. Поэтому для расчета поправок порядка $\varepsilon$ для данных собственных значений составим уравнение

$$
\Pi_{m_{0}, n} H+\varepsilon A_{n}(x) H=H B,
$$


в котором $H=H_{0}+\varepsilon H_{1}, H_{0}=\left[v_{k_{0}}(x), w_{k_{0}}(x)\right], H_{1}=\left[v_{k_{0}, 1}(x), w_{k_{0}, 1}(x)\right]$, $B=\varepsilon B_{0}$,

$$
B_{0}=\left(\begin{array}{ll}
b_{1} & b_{2} \\
\bar{b}_{2} & \bar{b}_{1}
\end{array}\right) .
$$

Приравнивая в (3.18) коэффициенты при $\varepsilon$, для $v_{k_{0}, 1}, w_{k_{0}, 1}$ получим аналогичные (3.10) линейные неоднородные уравнения, а неизвестные элементы матрицы (3.19) определим из условий разрешимости этих уравнений в классе функций вида $(3.12)$ (при $k=k_{0}$ ). Опуская соответствуюшие вычисления, аналогичные описанным в п. 2.2, приведем сразу окончательный результат:

$$
b_{1}=-d / 3, \quad b_{2}=-2 d / 3
$$

Из равенств (3.20) вытекает существование у матрицы (3.19) положительного собственного значения, что и доказывает неустойчивость всех циклов (3.3) при $l=1, n \notin S$. Отсюда косвенным образом следует, что по крайней мере в случае $l=1$ краевая задача (3.2) кроме циклов (3.3) должна иметь и другие аттракторы, нетривиально зависящие от обеих пространственных переменных.

Интересно то, что все результаты, полученные вьше при $l=1$, в полной мере сохраняются и в случае произвольного рационального значения $l^{2}$. Действительно, пусть $l^{2}=p_{0} / q_{0}$, где $p_{0}$ и $q_{0}$ взаимно просты. Рассмотрим, далее, множества номеров $n$

$$
\begin{aligned}
& S_{l}^{1}=\left\{n: n^{2} \neq k^{2}+m^{2} / l^{2}, k, m \in \mathbb{N}\right\} \\
& S_{l}^{2}=\left\{n: n^{2} \neq k^{2}+m^{2} l^{2}, k, m \in \mathbb{N}\right\}
\end{aligned}
$$

и предположим, к примеру, что $n \in S_{l}^{1}$. Тогда, повторяя практически дословно доказательство теоремы 3.1, убеждаемся в существовании такого не зависящего от $n$ достаточно малого $\varepsilon_{0}>0$, что при $0<\varepsilon \leqslant \varepsilon_{0}$ все циклы первого подсемейства (3.3) с номерами $n \in S_{l}^{1}$ равномерно экспоненщиально орбитально устойчивы (равномерность этого свойства вытекает из оценок $\left|k^{2}+m^{2} / l^{2}-n^{2}\right| \geqslant 1 / p_{0}$, гарантирующих отсутствие малых знаменателей в (3.13)). Если же номер $n$ таков, что $n^{2}=k_{0}^{2}+m_{0}^{2} / l^{2}$ при некоторых натуральных $k_{0}, m_{0}$, то сохраняются равенства (3.18), (3.19), а значит, рассматриваемый цикл оказывается неустойчивым. Отметим еще, что аналогичные выводы справедливы и для циклов второго подсемейства (3.3) при $n \in S_{l}^{2}$ и $n \notin S_{l}^{2}$ соответственно. И наконец, остается добавить, что поскольку каждое из множеств (3.21) содержит бесконечное число элементов, то в данном случае имеет место свойство гипербуферности.

Заканчивая исследование феномена гипербуферности для краевой задачи (3.2), предположим, что $l^{2}$ иррационально. Тогда условия (3.5) выполняются при всех $n$, а значит, теорема 3.1 гарантирует устойчивость при надлежашем уменьшении $\varepsilon$ любого конечного набора циклов (3.3). Иными словами, в этом случае наблюдается буферность. Вопрос о наличии здесь гипербуферности остается открытым и, по всей видимости, зависит от более тонких свойств числа $l^{2}$, а именно от скорости его аппроксимации рациональными числами. Для пояснения этого заметим, что при иррациональном $l^{2}$ могут существовать такие последовательности номеров $m_{s}, k_{s}, n_{s}, s \geqslant 1$, что, например,

$$
k_{s}^{2}+m_{s}^{2} / l^{2}-n_{s}^{2} \rightarrow 0, \quad s \rightarrow \infty
$$


Это означает, что во избежание малых знаменателей (см. (3.13)) алгоритм расчета асимптотики собственных значений краевой задачи (3.7) при $m=m_{s}, n=n_{s}$ на модах (3.9) с номерами $k=k_{s}$ следует проводить в форме (3.18), где

$$
B=\operatorname{diag}\left\{\lambda_{k_{s}}, \bar{\lambda}_{k_{s}}\right\}+\varepsilon B_{0} .
$$

Несложным подсчетом убеждаемся в том, что для матрицы $B_{0}$ из (3.23) справедливы прежние формулы (3.19), (3.20). Отсюда и из предельного равенства $\lim \lambda_{k_{s}}=0, s \rightarrow \infty$ (см. (3.22)), вытекает существование такого $s=s_{0}(\varepsilon)$, $\lim s_{0}(\varepsilon)=\infty, \varepsilon \rightarrow 0$, что при всех $s \geqslant s_{0}(\varepsilon)$ матрица (3.23) имеет собственное значение в полуплоскости $\{\lambda: \operatorname{Re}>0\}$. Тем самым, в случае (3.22) оказываются неустойчивыми циклы первого подсемейства (3.3) с номерами $n=n_{s}, s \geqslant s_{0}(\varepsilon)$.

Итак, установлено, что появление малых знаменателей в (3.13) приводит к неустойчивости соответствуюших циклов (3.3). Не ясно, однако, могут ли при этом оказаться неустойчивыми сразу все циклы (3.3) с достаточно большими номерами $n$.

3.2. Заключение. Рассмотрим уравнение (1.2) в произвольной ограниченной плоской области $\Omega$ и дополним его нулевыми граничными условиями Дирихле или Неймана на $\partial \Omega$. Из всей совокупности проделанных выше исследований можно вывести некоторый эвристический критерий наличия или отсутствия у получившейся краевой задачи свойства гипербуферности. Для его формулировки потребуется понятие спектральной щели:

$$
\nu(\Omega)=\inf _{\lambda_{n}, \lambda_{m}: \lambda_{n} \neq \lambda_{m}}\left|\lambda_{n}-\lambda_{m}\right|
$$

где $\lambda_{n}, n \geqslant 1,-$ собственные значения оператора $-\Delta$ в $\Omega$, соответствуюшие выбранным граничным условиям.

Упомянутый критерий состоит в следуюшем: если для области $\Omega$ выполняется условие $\nu(\Omega)>0$, то соответствуюшая краевая задача для уравнения (1.2) обладает свойством гипербуферности; если же $\nu(\Omega)=0$, то, вообше говоря, в такой области указанное свойство уравнения (1.2) отсутствует.

Для обоснования данного критерия в случае прямоугольника (3.1) заметим, что вне зависимости от граничных условий равенство $\nu(\Omega)=0$ эквивалентно требованию иррациональности $l^{2}$. Если же $l^{2}=p_{0} / q_{0}$ для некоторых натуральных $p_{0}, q_{0}$, то $\nu(\Omega) \geqslant \pi^{2} / p_{0}$. Таким образом, отсутствие феномена гипербуферности при $\nu(\Omega)=0$ иллюстрирует краевая задача (1.47), а наличие этого феномена при $\nu(\Omega)>0$ установлено вьше для краевых задач (1.8), (3.2).

В заключение обратим внимание на еще одну характерную особенность, а именно существенную зависимость аттракторов рассматриваемого уравнения при $\nu(\Omega)=0$ от граничных условий. Действительно, при иррациональном $l^{2}$ в рамках краевой задачи (3.2) наблюдается феномен буферности, в то время как у аналогичной краевой задачи с условиями Дирихле все циклы и торы оказываются неустойчивыми (см. п. 1.4). 


\section{Список литературы}

1. Колесов А. Ю., Мищенко Е.Ф., Розов Н.Х. Асимптотические методы исследования периодических решений нелинейных гиперболических уравнений // Тр. МИАН. 1998. T. 222 .

2. Колесов А. Ю., Мищенко Е.Ф., Розов Н.Х. Явление буферности в резонансных системах нелинейных гиперболических уравнений // УМН. 2000. Т. 55. № 2(332). С. 95-120.

3. Колесов А. Ю., Розов Н.X. Асимптотическая теория колебаний в системе Витта // Итоги науки и техники. Современная математика и ее прилож. Т. 67. М.: ВИНИТИ, 1999. C. $5-69$.

4. Колесов А. Ю., Розов Н.X. Явление буферности в RCLG-автогенераторе: теоретический анализ и результаты эксперимента // Тр. МИАН. 2001. Т. 233. С. 153-207.

5. Колесов А. Ю., Колесов Ю. С. Бифуркация автоколебаний сингулярно возмущенного волнового уравнения // ДАН СССР. 1990. Т. 315. № 2. С. 281-283.

6. Колесов А. Ю. Существование счетного числа устойчивых циклов в средах с дисперсией // Изв. РАН. Сер. матем. 1995. Т. 59. № 3. С. 141-158.

7. Лейбович C., Сибасс А. (ред.). Нелинейные волны. М.: Мир, 1977.

8. Cкотm Э. Волны в активных и нелинейных средах в приложении к электронике. М.: Советское радио, 1977.

9. Уизем Джс. Линейные и нелинейные волны. М.: Мир, 1977.

10. Ланда П. С. Нелинейные колебания и волны. М.: Физматлит, 1997.

11. Scheuer J., Malomed B. A. Stable and chaotic solutions of the complex Ginzburg-Landau equation with periodic boundary conditions // Physica D. 2002. V. 161. P. 102-115.

12. Захаров А.А., Колесов Ю.С. Пространственно неоднородные режимы в задаче хищник-жертва // Нелинейные колебания и экология. Ярославль. 1984. С. 3-15.

13. Колесов А. Ю., Розов Н. Х. Диффузионная буферность в одной математической модели биологии // Изв. РАН. Сер. матем. 1998. Т. 62. № 5. С. 135-164.

14. Боголюбов H. Н., Митропольский Ю. А. Асимптотические методы в теории нелинейных колебаний. М.: Наука, 1974.

15. Хенри Д. Геометрическая теория полулинейных параболических уравнений. М.: Мир, 1985.

16. Колесов Ю.С. Бифуркация инвариантных торов параболических систем с малой диффузией // Матем. сб. 1993. Т. 184. № 3. С. 121-136.

17. Колесов А. Ю., Розов H.X. Особенности динамики уравнения Гинзбурга-Ландау в плоской области // ТМФ. 2000. Т. 125. № 2. С. 205-220. 